\title{
Machine Learning for Arctic Sea Ice Physical Properties Estimation Using Dual-Polarimetric SAR Data
}

\author{
Katalin Blix, Martine M. Espeseth, Member, IEEE, Torbjørn Eltoft, Member, IEEE,
}

\begin{abstract}
This work introduces a novel method which combines Machine Learning (ML) techniques with dual-polarimetric (dual-pol) SAR observations for estimating quad-polarimetric (quad-pol) parameters, which are presumed to contain geophysical sea ice information. In the training phase, the output parameters are generated from quad-polarimetric observations obtained by Radarsat-2 (RS2), and the corresponding input data consists of features obtained from overlapping dual-pol Sentinel1 (S1) data. Then two, well recognized ML methods are studied to learn the functional relationship between the output and input data. These ML approaches are the Gaussian Process Regression (GPR) and Neural Network (NN) for regression models. The goal is to use the aforementioned ML techniques to generate Arctic sea ice information from freely available dual-pol observations acquired by S1, which can in general only be generated from quad-pol data.

Eight overlapping RS2 and S1 scenes were used to train and test the GPR and NN models. Statistical regression performance measures were computed to evaluate the strength of the ML regression methods. Then two scenes were selected for further evaluation, where overlapping optical images were available as well. This allowed the visual interpretation of the maps estimated by the ML models. Finally, one of the methods was tested on an entire S1 scene to perform prediction on areas outside of the RS2 and S1 overlap.

Our results indicate that the studied ML techniques can be utilized to increase the information retrieval capacity of the wide swath dual-pol S1 imagery, while embedding physical properties in the methodology.
\end{abstract}

Index Terms-Synthetic Aperture Radar, Sentinel-1, Radarsat2, Sea ice, Gaussian Process Regression, Neural Network, Polarimetry

\section{INTRODUCTION}

As a result of climate change, the extent and thickness of Arctic sea ice have been steadily declining for the last decades [1]. This makes it more susceptible to melting and more dynamic, and thus more difficult to monitor and forecast from remote sensing observations. Both from a climate perspective and due to the threat sea ice represents to human activities (ship traffic, fisheries. etc), it is getting even more important to develop reliable, high-resolution sea ice monitoring capabilities. Today, sea ice type maps are generated by experts at national ice centres based on interactive interpretation of synthetic aperture radar (SAR) satellite imagery, often

K. Blix, M. M. Espeseth, T. Eltoft are with the Department of Physics and Technology, UiT The Arctic University of Norway, 9037 Troms $\varnothing$, Norway.

Manuscript received Month XX, 201X; revised Month XX, 201X; accepted Month X, 201X. Date of publication Month XX, 201X; date of current version Month X, 201X. (Corresponding author: K. Blix (katalin.blix@uit.no)) supplemented by auxiliary data from other satellite sensors or surface observations [2].

In recent years, there have been made several scientific developments which have the potential to enable better sea ice monitoring. Significant achievements have been made in the field of SAR remote sensing, especially related to the use of polarisation diversity, a field commonly referred to as SAR or radar polarimetry [3], [4]. Furthermore, tremendous developments have taken place in the signal processing and Machine Learning (ML) domains [5]. In the computer science field, the capabilities in computation power and data storage have increased tremendously [6]. The objective of this paper is to explore the feasibility of using new powerful methods from the machine learning discipline to increase information extraction from dual polarisation SAR data, in particular with respect to sea ice characterisation.

SAR imagery is a key data source for generating sea ice maps. Its spatial characteristics are determined by the sea ice backscatter, and microwave radar signals are independent of light conditions, and hence available day and night, year around. However, SAR images of sea ice are difficult to translate into reliable sea ice maps. SAR signatures of sea ice are ambiguous as they depend on imaging geometry, season and weather condition, polarisation, frequency and angle of the incident radar signals, in addition to the actual ice type, with a potential snow cover on top. This makes the classification of SAR images into ice type maps very challenging. Even the binary discrimination between ice and open water is difficult due to varying radar intensities resulting from spatially variations in wind fields over the ocean.

It has long been acknowledged that coherent quadpolarimetric (quad-pol) SAR observations would improve the ability to retrieve geophysical properties of sea ice and reduce misinterpretations [7]. A quad-pol radar would simultaneously record all combinations of two orthogonal linear polarisations (most commonly horizontal and vertical), allowing for a complete characterisation of the backscattered electromagnetic vector field. Polarimetry has developed target decomposition methods, in which a received quad-pol signal is separated into contributions from different scattering mechanisms (e.g. [8]), as well as introduced new parameters and classification approaches, which have improved segmentation and classification performances (e.g. [9]-[11]). Some of the new quad-pol parameters have been found to be sensitive to specific surface properties such as roughness and salinity ( [8], [12]). Through the last three decades, many studies have also demonstrated 
the benefit of quad-pol data for sea ice characterisation [13][16].

Operational sea ice maps are however for the most part based on wide-swath SAR scenes, which generally are single or dual-polarisation data covering quite large surface areas. Sentinel-1 (S1) has been the primary work horse in this respect, since its launch in 2014. This mission comprises currently a constellation of two polar-orbiting satellites, working in a pre-programmed operation mode to provide regular and consistent C-band SAR image records on a free and open basis. This is the principal reason for why new ice charting methodologies should be targeting the use of S1 data modes.

In this work we study ML approaches to retrieve information from dual-polarimetric (dual-pol) data acquired by S1. ML techniques have been frequently used for sea ice classification purposes from SAR imagery. These methods include Neural Networks (NNs) [17]-[19], Support Vector Machines (SVM) [20], Bayesian classifiers [21] and Decision Trees (DT) [22]. These supervised ML approaches use SAR imagery for classifying sea ice scenes into ice type maps.

In this study, the objective is to use $M L$ for regression to learn the functional relationship between quad-pol output parameters and dual-pol input features. In the training phase, the quad-pol output parameters were generated from quad-pol data acquired by Radarsat-2 (RS2). We focus on two output parameters, which in previous studies have been shown to be associated with important sea ice properties, namely surface roughness and salinity. (See Sec. II for further details on the choice of these parameters.) The corresponding input data matrix was composed from coincident dual-pol observations from S1, and contained five or six input features. These dualpol input features are assumed to carry some information about the output parameters here (see Section III). The ML models learn the functional relationship between the output data generated from quad-pol observations and the corresponding input features composed of dual-pol data. Note, the dual-pol input matrix can also be generated from a subset of the quadpol Sinclair matrix, and the same ML regression can be applied to these data. This was successfully done in our previous work to perform feasibility study [23]. However, the overall goal here is to be able to use actual dual-pol observations to estimate the aforementioned output parameters by using ML regression, and eventually also extend the estimations to a complete S1 scenes. (We refer to [23] for the interested reader on those results.)

The investigated ML regression techniques were the Gaussian Process Regression (GPR) and a NN for regression models based on our previous results in [23]-[25]. These methods have previously been compared with other parametric and non-parametric models for remote sensing applications [26]-[28], and they were found to perform excellently for the given task. Both the GPR and NN were found to show good performance for learning the relationship between the output quad-pol parameters and the dual-pol input data. These methods are non-linear models, hence they do not rely on assumptions between the output and input data in contrast to other empirical algorithms. (Note that the combination of GPR and NN models have been successfully introduced for regional
Arctic sea ice extent predictions [29].)

Both the GPR and NN have different advantageous properties, besides their excellent regression strength. The GPR model proceeds by using Bayesian inversion, and hence providing the full posterior distribution of the estimates. This means that the variance of the estimated output is automatically provided. The variance represents a quantitative assessment of the certainty level of the estimates. The relative value of the certainty level reveals whether a new observation differs from the data used in the training process. This property is highly advantageous in practice. However, the GPR model requires the inversion of matrices, which is computationally expensive. Although there are several techniques, which allow to improve computational time, training a $\mathrm{NN}$ model requires less computational power.

In this work, we conducted a comprehensive study to evaluate the two ML approaches on overlapping quad-pol and dual-pol scenes. We used eight overlapping scenes with short time differences and similar incidence angles (IA). Two out of the eight scenes did also have optical image overlaps acquired by the Multi-Spectral Instrument (MSI) onboard the Sentinel2 satellite. This allowed the visual interpretation of the data and results. Seven overlaps had a deviation in IA equal or below two degrees. These seven scenes were used to establish the ML models for the two quad-pol output parameters. Then these models were tested on the eight scenes. Finally, these ML models were applied to large S1 scenes to perform the upscaling for the non-overlapping areas.In this work, up-scaling refers to the process of spatially extending the high-resolution, high informative quad-pol information to the larger lowresolution wide-swath dual-pol scenes. Hence, it involves both an up-scaling of information contents and a spatial extension. The statistical and visual results indicated that the ML models perform well to estimate quad-pol parameters from dual-pol data. The methodology opens the possibility for spatial upscaling of quad-pol information by using large scale dual-pol imagery, and hence increasing the capacity of using dual-pol Sentinel-1 observations for sea ice monitoring.

The rest of this work is organized as follows. Section II provides on overview on the theory of polarimetry, Section III describes the data used in this study, Section IV discusses the methodology, Section V presents and discusses the results, and finally Section VI concludes this work.

\section{POLARIMETRY: TWO POLARIMETRIC PARAMETERS}

Radar backscattering from sea ice is a function of both physical characteristics like surface roughness, which is resulting from the ice dynamics, and electric characteristics like the dielectric constant, which is related to humidity and salinity contents. Since ice types, at least to some degree, are defined by roughness and dielectric properties, it is expected that ice type classification would benefit from using input parameters that are sensitive to these geophysical properties. In this feasibility study, we have chosen to perform dual-toquad-pol regression prediction analysis on two polarimetric parameters which have previously been found to be sensitive to roughness and dielectric properties, namely the circular correlation coefficient, $\rho_{R R L L}$ and the linear co-pol power ratio, 
$R_{V V H H}$. These two parameters have been proven valuable for sea ice classification in e.g. [30] and [31]. They are further discussed below.

\section{A. The circular correlation coefficient}

The circular correlation coefficient is defined as

$$
\rho_{R R L L}=\frac{\left\langle S_{R R} S_{L L}^{*}\right\rangle}{\sqrt{\left\langle S_{R R} S_{R R}^{*}\right\rangle\left\langle S_{L L} S_{L L}^{*}\right\rangle}},
$$

where $S_{R R}$ and $S_{L L}$ are the scattering coefficients in righthand circular (RHC) and left-hand circular (LHC) polarisations, respectively. These are related to the scattering coefficients in the linear vertical $(\mathrm{V})$ and horizontal $(\mathrm{H})$ polarisations by [4]:

$$
\begin{gathered}
S_{R R}=S_{V V}-S_{H H}+2 j S_{H V}, \\
S_{L L}=S_{H H}-S_{V V}+2 j S_{H V},
\end{gathered}
$$

where the first subscript indicates transmit and the second is receive. For a RHC polarised radar wave incident on a smooth surface, the reflected wave can analytically be determined to be LHC if the IA is below Brewster's angle, and RHC if it is above [32]. For a mono-static radar, which measures the backscattered signal, simulation studies conducted by the authors of [32] show that at low to medium IA the LHC component dominates the signal for RHC incidence, and that this dominance decreases with increasing incidence angle and roughness. The complete opposite is the case for LHC incidence. Hence, the circular backscatter returns are strongly coupled to the surface roughness. In [33], the authors investigated the relationship between several polarimetric parameters extracted from multifrequency SAR data and surface roughness, and found an enhanced sensitivity of $\rho_{R R L L}$. This finding has been confirmed by several authors. Schuler et al. [34] derived a theoretical relationship between $\rho_{R R L L}$ and roughness using a simple scattering model, which in later publications is denoted the X-Bragg model (e.g. [4]). The scattering is assumed to be Bragg-scattering from tilted planes, and the large-scale roughness is characterised in terms of a slope-induced distribution of the polarimetric orientation angle. It is noted that in this model the circular correlation coefficient is independent of dielectric constant, but dependent on IA through the dependency of polarimetric orientation angle on IA [34] (see also [35] for more details on the dependency of the $\rho_{R R L L}$ on the orientation angle). In [30], the authors concluded that $\rho_{R R L L}$ is a good parameter for discrimination between different sea ice types.

\section{B. The linear co-polarisation power ratio}

Another polarisation parameter that has been widely studied in relation to sea ice characterisation from polarimetric measurements, is the linear co-polarisation power ratio or simply the co-pol ratio, i.e.

$$
R_{H H V V}=\frac{\left\langle S_{H H} S_{H H}^{*}\right\rangle}{\left\langle S_{V V} S_{V V}^{*}\right\rangle} .
$$

In some surface scattering models, like the small perturbation model (or the Bragg model) and the composite model (or twoscale models) [36], it follows explicitly from the theoretical expressions that the co-pol ratio is a function of the dielectric constant and IA, but independent of the small-scale roughness. This property has been used to estimate surface humidity from polarimetric measurements in e.g. [12]. Even though these models are not generally applicable for describing backscatter from sea ice, they give good predictions for certain ice types (e.g., smooth, newly frozen ice), especially at longer wavelengths (L- and P-band) [37]. Also an empirical model, like the Oh model [38] predicts that the co-pol ratio is directly sensitive to the dielectric property of the medium. Several analyses have found evidence that $R_{H H V V}$ can discriminate between new ice and open water [7], and it is also useful for separating thin sea ice and smooth first-year ice [31]. The co-pol ratio depends on the IA, and with $\mathrm{HH} / \mathrm{VV}$, this ratio will in general decrease with increasing IA. Note that, the IA slope varies depending on the observed surface. The co-pol ratio seems to be most useful at longer wavelengths (L-band and P-band), but there are studies which show clear benefit also at C-band, see e.g., [31]. This is the reason why we have chosen to include $R_{H H V V}$ in our analysis.

\section{Polarimetric ML regression}

As noted from Eqs. (1) to (4), it is not possible to compute $\rho_{R R L L}$ and $R_{H H V V}$ directly from the dual-pol input $\mathrm{S} 1$ data, as all the needed observations are not available. In our work, we attempt to predict the absolute value of the circular correlation coefficient, i.e. $\left|\rho_{R R L L}\right|$, and the co-pol ratio $R_{H H V V}$ from input parameters that can be generated from dual-pol observations. The approach is to use supervised learning methodologies to train ML regression models to learn the functional relationship between the dual-pol input parameters and the quad-pol output parameters. In general, this training is performed on a training set $D=\{\mathbf{y}, \mathbf{X}\}$, where in our case $\mathbf{y}$ is a $(1 \times N)$ vector of quad-pol output samples calculated from RS2 scenes, and $\mathbf{X}$ is a $(k \times N)$ matrix of $N$ $k$-dimensional dual-pol input parameters calculated from accurately co-registered $\mathrm{S} 1$ scenes. Hence, an estimated functional relationship between $\mathbf{y}$ and $\mathbf{X}$, i.e. $\mathbf{y}=\hat{f}(\mathbf{X})$, is realized by the trained ML-models, and subsequently used to predict the output quad-pol parameters. The details are given in section III and section IV below.

\section{DATA}

\section{A. Data acquisition}

In total, we used eight overlapping RS2 and S1 scenes with a very short time difference of less than 40 minutes. The sensor properties of these two satellites and the modes used are shown in Table I. The S1 scenes have a much larger coverage than the fine-quad-pol mode of RS2, whereas the pixel spacing is much finer for RS2 than S1. All the scenes were acquired between July and September in the Arctic, i.e., during melt-season. The scenes acquired 25/08 and 03/09 are related to the study area investigated in [39].

The location and description of these overlaps can be seen in Fig. 1 and Table II. Note that, the entire S1 scenes are not shown, only the overlapping parts. The spatial extent of the scenes covers a large area, and they include various types of 
sea ice and percentage of ice concentration. For training the ML models, we chose overlaps with a $2^{\circ}$ maximum difference in IA. These overlaps correspond to scenes number 1 to 7 . An additional overlap was included for testing the performance of the ML models, when the difference in IA is larger than $2^{\circ}$. This is scene number 8 , and indicated with a star symbol in Table II.

TABLE I

PROPERTIES OF THE SAR SENSORS INVESTIGATED IN THIS STUDY, I.E., SENTINEL-1 (S1) [40] AND RADARSAT-2 (RS2) [41]). S1 PRODUCTS USED ARE GRD (GROUND RANGE DETECTED PRODUCTS) HR (HIGH RESOLUTION). THE RS2 RANGE PIXEL SPACING IS GIVEN IN SLANT RANGE, WHEREAS S1 RANGE PIXEL SPACING IS IN GROUND RANGE. PIXEL SPACING IS IN RNG. $\times$ AZ.

\begin{tabular}{|l||cc||}
\hline \hline & Radarsat-2 & Sentinel-1 \\
\hline \hline Mode & Fine Quad-pol (FQ) & Extra-wide Swath Mode (EW) \\
\hline Polarization & HH, HV, VH, VV & HH, HV \\
\hline Pixel spacing & $4.7 \times 5.1 \mathrm{~m}$ & $25 \times 25 \mathrm{~m}$ \\
\hline Swath width & $25 \mathrm{~km}$ & $410 \mathrm{~km}$ \\
\hline NESZ & $-35 \pm 4 \mathrm{~dB}$ & $<-23.1 \mathrm{~dB}$ \\
\hline \hline
\end{tabular}

All the scenes are radiometrically calibrated (see [42] for RS2 and [40] for S1), and the RS2 parameters are produced within $9 \times 9$ averaging windows. Then the investigated features are calculated from S1 and RS2 products and these are geo-coded to a spatial common grid with equal number of pixels on the ground covering approximately $50 \times 50 \mathrm{~m}$. The time difference between $\mathrm{S} 1$ and RS2 scenes are small (see Table II), but a co-registration (linear shift of the pixels in S1) is performed on the geo-coded products to adjust for the minor sea ice drift between the two scenes. Following this procedure, each S1 and RS2 pair (scene nr. in Table II) represents a stack of features covering the same area, similar pixel spacing, but some deviations in the level is smoothing are expected due to different initial spatial resolution (see Table I).

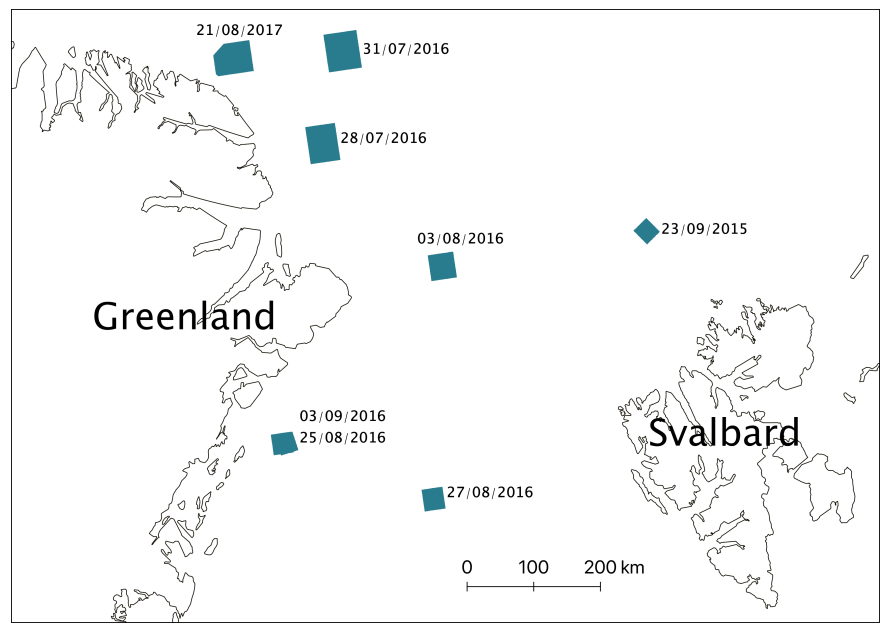

Fig. 1. Study area of the eight overlapping Radarsat-2 and Sentinel-1 scenes used for training, testing, and validation. Only the overlapping parts of Sentinel-1 scenes are shown, not the entire scene.

\section{B. Polarimetric data}

The two studied quad-pol output parameters, henceforth denoted $y^{1}$ and $y^{2}$ were generated from the RS2 data and they
TABLE II

DESCRIPTION OF THE EIGHT SCENES: SCENE NUMBER, DATE, ACQUISITION TIME AND THE MEAN INCIDENT ANGLE (IA) FOR RADARSAT-2 (RS2) AND SENTINEL-1 (S1). ONLY THE MEAN INCIDENT ANGLE OF THE OVERLAPPING AREA FOR S1 IS PROVIDED. THE INCIDENCE ANGLE RANGE OF THE SCANSAR MODE OF SENTINEL-1 IS 30-45 DEGREES [40].

\begin{tabular}{|c||c|c|c||c|c||}
\hline \hline Scene nr. & Date & Time RS2 & Time S1 & IA RS2 & IA S1 \\
\hline \hline 1 & $23 / 09 / 2015$ & $15: 19$ & $15: 13$ & $40.2-41.6$ & 41 \\
\hline 2 & $28 / 07 / 2016$ & $10: 07$ & $09: 55$ & $30.7-33.7$ & 34 \\
\hline 3 & $31 / 07 / 2016$ & $13: 40$ & $13: 36$ & $26.2-29.4$ & 27 \\
\hline 4 & $03 / 08 / 2016$ & $15: 32$ & $15: 37$ & $27.0-28.8$ & 29 \\
\hline 5 & $27 / 08 / 2016$ & $17: 11$ & $17: 15$ & $40.3-41.7$ & 43 \\
\hline 6 & $03 / 09 / 2016$ & $17: 07$ & $17: 07$ & $28.1-29.9$ & 29 \\
\hline 7 & $21 / 08 / 2017$ & $16: 02$ & $15: 46$ & $26.2-29.5$ & 26 \\
\hline \hline $8^{\star}$ & $25 / 08 / 2016$ & $18: 10$ & $17: 32$ & $44.5-45.7$ & 36 \\
\hline
\end{tabular}

provide information about the sea ice roughness and dielectric properties, respectively. They can be expressed by

$$
\begin{gathered}
y^{1}=\left|\left\langle S_{R R} S_{L L}^{\star}\right\rangle\right| / \sqrt{\left\langle\left|S_{R R}\right|^{2}\right\rangle\left\langle\left|S_{L L}\right|^{2}\right\rangle} \\
y^{2} \\
=\left\langle\left|S_{H H}\right|^{2}\right\rangle /\left\langle\left|S_{V V}\right|^{2}\right\rangle,
\end{gathered}
$$

where $y^{1}=\left|\rho_{R R L L}\right|$ (discussed in section II-A), and $y^{2}=$ $R_{H H V V}$ (discussed in section II-B). Speckle noise was reduced by using spatial averaging, and it is denoted by $\langle\cdot\rangle$.

The input data was generated from the dual-pol S1 covariance matrix $\mathbf{C}$, which can be expressed by

$$
\mathbf{C}=\left[\begin{array}{ll}
C_{11} & C_{12} \\
C_{12} & C_{22}
\end{array}\right] .
$$

We used a dual-pol system $C$ that transmits on the horizontal (H) channel and receives on horizontal and vertical (V) polarization channels. The dual-pol S1 system $C$ can be written by

$$
\mathbf{C}=\left\langle\left[\begin{array}{cc}
\left|S_{H H}\right|^{2} & S_{H H} S_{H V}^{\star} \\
S_{H V} S_{H H}^{\star} & \left|S_{H V}\right|^{2}
\end{array}\right]\right\rangle .
$$

This dual-pol system $C$ was used to generate five features denoted by $f_{1}, \ldots, f_{5}$ to build the $5 \times N$ dimensional $\mathrm{S} 1$ input matrix $\mathbf{X}$, where $N$ is the total number of pixels. These five features are defined in Eq.(7). Note that, for assessing the possibilities of improving model performance by also taking into account the IA, an extended input matrix $\mathbf{X}$ was composed, which included IA as a sixth feature.

$$
\left[\begin{array}{l}
f_{1} \\
f_{2} \\
f_{3} \\
f_{4} \\
f_{5} \\
f_{6}
\end{array}\right]=\left[\begin{array}{c}
10 \log _{10}\left(C_{11}\right) \\
10 \log _{10}\left(C_{22}\right) \\
C_{11} / C_{22} \\
C_{11}-C_{22} \\
\left(C_{11}-C_{22}\right) /\left(C_{11}+C_{22}\right) \\
\text { IA }
\end{array}\right] .
$$

Input feature $f_{1}$ and $f_{2}$ are only based on single polarization channels, namely $\mathrm{HH}$ and HV, respectively. These features, mostly the HH intensity, have been used in several sea ice studies (see e.g., [43]-[45]). The construction of the input features presented in Eq. (7) considers both ratio- and nonratio based features, sum and difference between features, and single- and dual-polarization features. In this way, we aim to limit the polarimetric information loss in our selection of 
the features from the dual-polarimteric data, and at the same time, maximize the relevant polarimetric information needed to describe the output parameters. This physical relationship was learnt by using the ML methods.

Then the output (RS2) and input (S1) data that were used for training and testing the performance of the ML models is $D=\left\{\mathbf{y}^{i}, \mathbf{X}\right\}$, for $i=1,2$.

Figure 2 shows scatterplots of the generated output parameter $\mathrm{y}^{1}$ (top-row) and $\mathrm{y}^{2}$ (bottom-row) versus the first five input features for the training data for RS2. The gray markers represent the values from the entire RS2 scene acquired 3/9/2016. It can be seen that there is some structured relationship between the input features and output parameters, and not complete randomness. The functional dependency between the output parameters and input features show large variations.

The scatterplots also reveal that output parameter $y^{2}$ has a stronger dependency on the input features than output parameter $\mathrm{y}^{1}$.

Note that all experiments were conducted two times; once with features $f_{1}, \cdots f_{6}$ as input, and once with features $f_{1}, \cdots f_{5}$.

\section{METHOD}

\section{A. Experimental setup}

We used the seven overlapping scenes (scene number $1, \ldots, 7$ ) for training the ML models. The training data was obtained by randomly sampling all the seven output-input data to create a representative training data. The number of observations for the training data was 1084 . Then eight ML models were established. These were the Gaussian Process Regression (GPR) and Neural Network (NN) for output parameter $y^{1}$ and $y^{2}$, and for the five (without IA) and six (with IA) dimensional input data matrix. Then all the eight models were evaluated on the eight individual scenes. (The 8th scene is the additional one, including large differences in IA.) The size of the test data varied between $4 \times 10^{5}$ and $10^{6}$. Model performance was assessed by computing statistical performance measures, the Normalized Root Mean Squared Error (NRMSE), the Mean Absolute Error (MAE) and the Pearson correlation coefficient $\left(\mathrm{R}^{2}\right)$. These measures can be written by

$$
\begin{aligned}
\text { NRMSE } & =\frac{1}{y_{\max }-y_{\min }} \sqrt{\frac{1}{N} \sum_{i=1}^{N}\left(y_{i}-\hat{y}_{i}\right)^{2}} \\
\text { MAE } & =\frac{1}{N} \sum_{i=1}^{N}\left|\left(y_{i}-\hat{y}_{i}\right)\right| \\
\mathrm{R}^{2} & =\frac{\sum_{i=1}^{N}\left(\hat{y}_{i}-\bar{y}\right)^{2}}{\sum_{i=1}^{N}\left(y_{i}-\bar{y}_{i}\right)^{2}},
\end{aligned}
$$

where $N$ is the number of observations in the test set, $y$ is the generated quad-pol output from RS2, $\hat{y}$ is the estimated quad-pol output from S1, $y_{\max }$ is the maximum value, $y_{\min }$ is the minimum value, and $\bar{y}$ is the mean value in the test set. Finally, up-scaling was performed for the eight large S1 scenes $\left(\mathbf{X}^{\prime}\right)$. Fig. 3 shows the flowchart of the approach.

In this work we used the software MatLab for implementing the machine learning approaches and producing the results,
SNAP (Sentinel toolbox) for calibration, GDAL and Python for geo-coding and co-registration.

\section{B. Machine Learning methods}

We chose to study two ML approaches based on our earlier results [23]. These were the GPR and NN. This section provides a brief overview on the ML models.

1) Gaussian Process Regression: The GPR is a flexible semi-analytical Bayesian approach, using non-linear mapping to relate the output $\mathbf{y}^{i}, i=1,2$ to the input $\mathbf{X}$. This can be expressed by $\mathbf{y}=f(\mathbf{X})+\varepsilon$, where the term $\varepsilon$ is independently, identically Gaussian distributed noise, with zero mean and constant variance. Prediction is done by placing a multivariate joint Gaussian distribution over the function values allowing to analytically derive the posterior distribution of the predicted output [46]. Then the posterior distribution of a new output $\mathbf{y}^{\prime}$ is

$$
\begin{aligned}
p\left(\mathbf{y}^{\prime} \mid \mathbf{X}^{\prime}, \mathcal{D}\right) & =\mathcal{N}\left(\mathbf{y}^{\prime} \mid \mu_{\mathrm{GP}^{\prime}}, \sigma_{\mathrm{GP}^{\prime}}^{2}\right) \\
\boldsymbol{\mu}_{\mathrm{GP}^{\prime}} & =\mathbf{k}_{\mathbf{f}^{\prime}}^{\top}\left(\mathbf{K}_{\mathrm{ff}}+\boldsymbol{\sigma}^{2} \mathbf{I}_{n}\right)^{-1} \mathbf{y}=\mathbf{k}_{\mathbf{f}^{\prime}}^{\top} \boldsymbol{\alpha} \\
\boldsymbol{\sigma}_{\mathrm{GP}^{\prime}} & =\sigma^{2}+k_{\prime \prime}-\mathbf{k}_{\mathbf{f}^{\prime}}^{\top}\left(\mathbf{K}_{\mathrm{ff}}+\sigma^{2} \mathbf{I}_{n}\right)^{-1} \mathbf{k}_{\mathbf{f}^{\prime}},
\end{aligned}
$$

where $\boldsymbol{\mu}_{\mathrm{GP}^{\prime}}$ is the predicted output, $\boldsymbol{\sigma}_{\mathrm{GP}^{\prime}}$ is the variance, $\mathbf{k}_{\mathbf{f}^{\prime}}$ is the covariance between the training vector and the test point, $\boldsymbol{\alpha}=\left(\mathbf{K}_{\mathrm{ff}}+\sigma^{2} \mathbf{I}_{n}\right)^{-1} \mathbf{y}$ is the weight vector and $k_{\prime \prime}$ is the covariance between the test point with itself. The elements of the covariance matrices are computed by using a kernel function. In this work, several kernel functions were evaluated, and it was found that the anisotropic Gaussian kernel is the most suitable, which is in good correspondence with earlier results. This can be expressed by

$$
k\left(X_{m}, X_{n}\right)=\nu^{2} \exp \left(-\frac{1}{2} \sum_{d=1}^{D}\left(\frac{X_{m}^{d}-X_{n}^{d}}{\lambda_{d}}\right)^{2}\right),
$$

where the hyper-parameters are $\lambda_{d}$ and $\nu$, and they are the length-scale and scaling-factor for the five or six dimension $d$. The hyper-parameters were optimized through the logmarginal likelihood by using gradient descent method.

2) Neural Network: The second ML regression method is a Multi Layer Perceptron (MLP) neural network (NN). Th MLP had one hidden layer and 25 neurons [25]. For training the NN, the Levenberg - Marquardt (L-M) algorithm was used, and the activation function was hyperbolic tangent sigmoid function. Training, testing and validation was done by using $70 \%$, $15 \%$ and $15 \%$ of the training data for learning, testing and validation, respectively.

\section{Results And Discussions}

\section{A. Overlapping areas}

Tables III and IV summarize the results of the estimated output parameters for the eight scenes, when IA was excluded and included in the input data, respectively. Including IA as a sixth feature in the input data has not resulted in significant improvements in the computed statistical regression performance measures (Table IV). This is visualized in Figure 4. The red markers correspond to the results when IA was not 

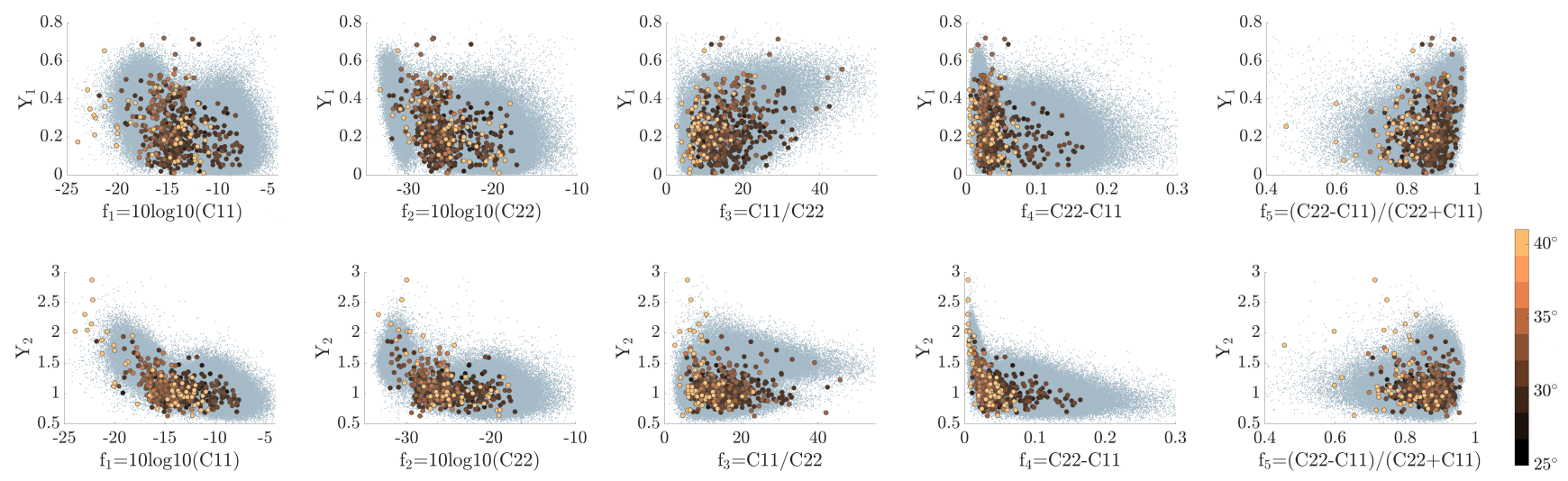

Fig. 2. Scatterplots of $y^{1}$ and $y^{2}$ versus the input features $\left(f_{1}, \ldots, f_{5}\right)$ from Radarsat- 2 . The values are selected from the training set, and contain various incidence angles (indicated by the brown gradients, see legend to the right) and sea ice conditions. The gray markers are values from the entire Radarsat- 2 scene acquired 3/9/2016.
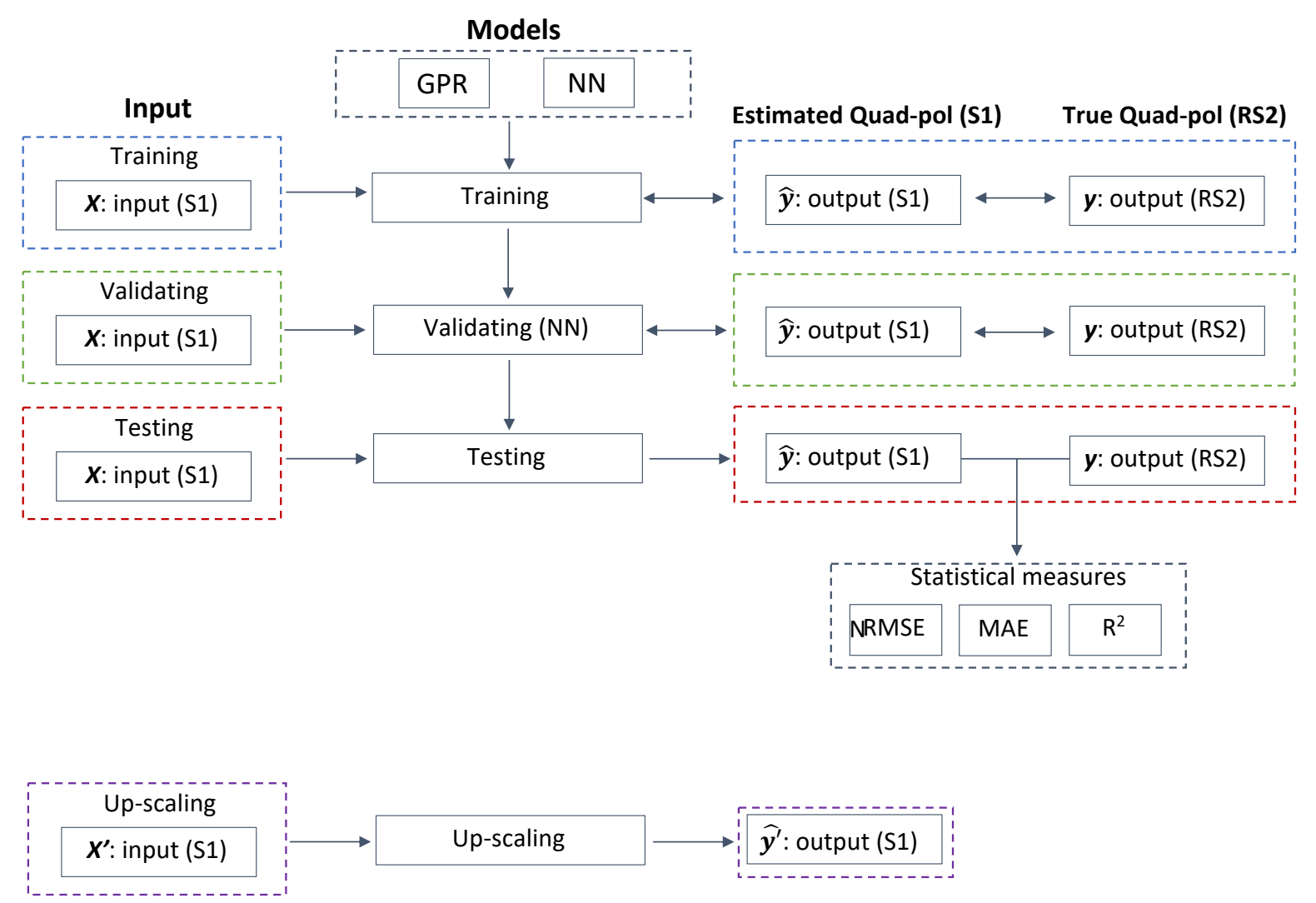

Fig. 3. Flowchart of the different steps involving the data input, the two models tested, training, testing, validation and up-scaling.

included, and the blue ones, when IA was included. The circles and squares represent the GPR and NN, respectively. These plots clearly demonstrate that including IA as the sixth feature in the input data has not resulted in significant improvements in the computed statistical measures. This is especially the case for output parameter $\mathrm{y}^{2}$. Therefore, in the rest of this paper only those results are presented and discussed, when IA was not included in the input data.

The computed statistical measures in Table III show a stronger performance for the co-pol ratio, $\mathrm{y}^{2}$, which is expected to carry information about the dielectric property or salinity of sea ice, than for $y^{1}$, the circular correlation coefficient, which is expected to be correlate with roughness. Note that a high value of $y^{2}$ would be associated with a high dielectric constant, as in water, whereas a high value of $y^{1}$ would be associated with a smooth surface. The observed strong performance for $\mathrm{y}^{2}$ might confirm the fact that the output co-pol ratio has a stronger dependency of the input features. This can also be observed in the scatterplots presented in Fig. 2. In general, for $y^{2}$ the computed NRMSE and MAE values are low and the $\mathrm{R}^{2}$ values are high, indicating an outstanding performance. 
TABLE III

COMPUTED STATISTICAL MEASURES FOR THE MACHINE LEARNING MODELS FOR THE EIGHT OVERLAPPING SENTINEL-1 SCENES. INCIDENT ANGLE WAS NOT INCLUDED IN THE INPUT FEATURES

\begin{tabular}{|c||c|c|c||c|c|c||}
\hline \multicolumn{1}{|c||}{ Scene 1 } & \multicolumn{3}{c||}{$y^{1}$} & \multicolumn{3}{c||}{$y^{2}$} \\
\hline \hline Model & NRMSE & MAE & $\mathrm{R}^{2}$ & NRMSE & MAE & $\mathrm{R}^{2}$ \\
\hline GPR & 0.0799 & 0.0316 & 0.6889 & 0.0409 & 0.0768 & 0.8862 \\
\hline NN & 0.0812 & 0.0382 & 0.6778 & 0.0423 & 0.0833 & 0.8854 \\
\hline \hline \multicolumn{1}{|c||}{ Scene 2 } & \multicolumn{3}{|c||}{$y^{1}$} & \multicolumn{3}{c||}{$y^{2}$} \\
\hline \hline Model & NRMSE & MAE & $\mathrm{R}^{2}$ & NRMSE & MAE & $\mathrm{R}^{2}$ \\
\hline GPR & 0.1259 & 0.0722 & 0.6404 & 0.0669 & 0.1118 & 0.9059 \\
\hline NN & 0.1247 & 0.0761 & 0.6421 & 0.0671 & 0.1112 & 0.9062 \\
\hline \hline Scene 3 & \multicolumn{5}{|c||}{$y^{1}$} & \multicolumn{3}{c||}{$y^{2}$} \\
\hline \hline Model & NRMSE & MAE & $\mathrm{R}^{2}$ & NRMSE & MAE & $\mathrm{R}^{2}$ \\
\hline GPR & 0.0934 & 0.0510 & 0.5916 & 0.0585 & 0.0770 & 0.9116 \\
\hline NN & 0.0964 & 0.0578 & 0.5667 & 0.0584 & 0.0767 & 0.9158 \\
\hline \hline Scene 4 & \multicolumn{5}{|c||}{$y^{1}$} & \multicolumn{3}{|c||}{$y^{2}$} \\
\hline \hline Model & NRMSE & MAE & $\mathrm{R}^{2}$ & NRMSE & MAE & $\mathrm{R}^{2}$ \\
\hline GPR & 0.1027 & 0.0487 & 0.6321 & 0.0613 & 0.0806 & 0.9346 \\
\hline NN & 0.1080 & 0.0558 & 0.6041 & 0.0581 & 0.0760 & 0.9410 \\
\hline \hline Scene 5 & \multicolumn{5}{|c||}{$y^{1}$} & \multicolumn{3}{c||}{$y^{2}$} \\
\hline \hline Model & NRMSE & MAE & $\mathrm{R}^{2}$ & NRMSE & MAE & $\mathrm{R}^{2}$ \\
\hline GPR & 0.1130 & 0.0755 & 0.5555 & 0.0856 & 0.2690 & 0.7436 \\
\hline NN & 0.1157 & 0.0796 & 0.5410 & 0.0869 & 0.2658 & 0.7350 \\
\hline \hline Scene 6 & \multicolumn{5}{|c||}{$y^{1}$} & \multicolumn{3}{c||}{$y^{2}$} \\
\hline \hline Model & NRMSE & MAE & $\mathrm{R}^{2}$ & NRMSE & MAE & $\mathrm{R}^{2}$ \\
\hline GPR & 0.1197 & 0.0654 & 0.5634 & 0.0594 & 0.1272 & 0.8579 \\
\hline NN & 0.1286 & 0.0708 & 0.4277 & 0.0558 & 0.1203 & 0.8779 \\
\hline \hline Scene 7 & \multicolumn{5}{|c||}{$y^{1}$} & \multicolumn{3}{c|}{$y^{2}$} \\
\hline \hline Model & NRMSE & MAE & $\mathrm{R}^{2}$ & NRMSE & MAE & $\mathrm{R}^{2}$ \\
\hline GPR & 0.1091 & 0.0610 & 0.3982 & 0.0546 & 0.0817 & 0.9310 \\
\hline NN & 0.1097 & 0.0654 & 0.3952 & 0.0546 & 0.0851 & 0.9349 \\
\hline \hline Scene 8 & \multicolumn{3}{|c||}{$y^{1}$} & \multicolumn{3}{c|}{$y^{2}$} \\
\hline \hline Model & NRMSE & MAE & $\mathrm{R}^{2}$ & NRMSE & MAE & $\mathrm{R}^{2}$ \\
\hline GPR & 0.1131 & 0.0789 & 0.2914 & 0.0714 & 0.1990 & 0.6482 \\
\hline NN & 0.1127 & 0.0805 & 0.2930 & 0.0733 & 0.2092 & 0.6298 \\
\hline \hline
\end{tabular}

It can also be observed that scene 8 shows a slightly decreased regression strength. Note, there were no datapoints from scene 8 included in the training set because of the larger deviation in IA between the RS2 and S1 scenes for this overlap. However, the computed statistical regression performance measures show acceptable regression strength, and hence demonstrating that both ML models have excellent generalization capabilities.

It is also observed from Tables III and IV that the GPR and NN showed equally strong performance based on the computed statistical measures.

For visually illustrating the results, two scenes were chosen for a more detailed analysis. These were the ones where overlapping optical images from Sentinel-2 (S2) were available as well. Figs. 5, 6, 7 and 8 show the scenes and results for $y^{1}$ (left columns) and $\mathrm{y}^{2}$ (right columns) for these scenes. In Figs. 5 and 7 the top rows show the true RGB images from S2. The RS2-S1 overlaps are indicated with the black squares. It can be seen that both scenes include many sea ice types, including young and/or first-year ice presumably thin- and thicker ice, and leads and open water. See [39] for a through description of the sea ice conditions in this area during the same time period. The second rows show the $\mathrm{HH}$ intensity images.

In Figs. 5 and 7 the top rows are the generated output
TABLE IV

COMPUTED STATISTICAL MEASURES FOR THE MACHINE LEARNING MODELS FOR THE EIGHT OVERLAPPING SENTINEL-1 SCENES. INCIDENT ANGLE WAS INCLUDED IN THE INPUT FEATURES.

\begin{tabular}{|c|c|c|c|c|c|c|}
\hline Scene 1 & \multicolumn{3}{|c|}{$y^{1}$} & \multicolumn{3}{|c|}{$y^{2}$} \\
\hline Model & NRMSE & MAE & $\mathrm{R}^{2}$ & NRMSE & MAE & $\overline{\mathrm{R}^{2}}$ \\
\hline GPR & 0.0859 & 0.0350 & \begin{tabular}{|l|l}
0.7089 \\
\end{tabular} & 0.0410 & \begin{tabular}{|l|}
0.0764 \\
\end{tabular} & 0.8854 \\
\hline $\mathrm{NN}$ & 0.0863 & \begin{tabular}{|l|l|}
0.0349 \\
\end{tabular} & 0.6920 & 0.0411 & \begin{tabular}{|l|l|}
0.0793 \\
\end{tabular} & 0.8873 \\
\hline Scene 2 & \multicolumn{3}{|c|}{$y^{1}$} & \multicolumn{3}{|c|}{$y^{2}$} \\
\hline Model & NRMSE & MAE & $\mathrm{R}^{2}$ & NRMSE & MAE & $\mathrm{R}^{2}$ \\
\hline GPR & 0.1238 & 0.0710 & 0.6595 & 0.0670 & \begin{tabular}{|l|l|}
0.1119 \\
\end{tabular} & 0.9055 \\
\hline $\mathrm{NN}$ & 0.1189 & 0.0686 & 0.6696 & 0.0686 & \begin{tabular}{|l|}
0.1131 \\
\end{tabular} & 0.9082 \\
\hline Scene 3 & \multicolumn{3}{|c|}{$y^{1}$} & \multicolumn{3}{|c|}{$y^{2}$} \\
\hline Model & NRMSE & MAE & $\mathrm{R}^{2}$ & NRMSE & MAE & $\mathrm{R}^{2}$ \\
\hline GPR & 0.0930 & \begin{tabular}{|l|}
0.0502 \\
\end{tabular} & \begin{tabular}{|l|}
0.5944 \\
\end{tabular} & 0.0588 & \begin{tabular}{|l|}
0.0776 \\
\end{tabular} & 0.9113 \\
\hline NN & 0.0925 & 0.0493 & \begin{tabular}{|l|}
0.6112 \\
\end{tabular} & 0.0565 & \begin{tabular}{|l|l|} 
\\
\end{tabular} & 0.9161 \\
\hline Scene 4 & \multicolumn{3}{|c|}{$y^{1}$} & \multicolumn{3}{|c|}{$y^{2}$} \\
\hline Model & NRMSE & MAE & $\mathrm{R}^{2}$ & NRMSE & MAE & $\mathrm{R}^{2}$ \\
\hline GPR & 0.1005 & 0.0471 & \begin{tabular}{|l}
0.6304 \\
\end{tabular} & 0.0616 & 0.0811 & 0.9344 \\
\hline NN & 0.0991 & \begin{tabular}{|l|l|}
0.0463 \\
\end{tabular} & 0.6358 & 0.0573 & \begin{tabular}{|l|}
0.0729 \\
\end{tabular} & 0.9424 \\
\hline Scene 5 & \multicolumn{3}{|c|}{$y^{1}$} & \multicolumn{3}{|c|}{$y^{2}$} \\
\hline Model & NRMSE & MAE & $\mathrm{R}^{2}$ & NRMSE & MAE & $\mathrm{R}^{2}$ \\
\hline GPR & 0.1114 & \begin{tabular}{|l}
0.0747 \\
\end{tabular} & 0.5722 & 0.0854 & 0.2665 & 0.7450 \\
\hline NN & 0.1133 & \begin{tabular}{|l|l|}
0.0759 \\
\end{tabular} & \begin{tabular}{|l|l|}
0.5536 \\
\end{tabular} & 0.0868 & 0.2655 & 0.7358 \\
\hline Scene 6 & \multicolumn{3}{|c|}{$y^{1}$} & \multicolumn{3}{|c|}{$y^{2}$} \\
\hline Model & NRMSE & MAE & $\overline{\mathrm{R}^{2}}$ & NRMSE & $\overline{\text { MAE }}$ & $\mathrm{R}^{2}$ \\
\hline GPR & 0.1267 & 0.0689 & \begin{tabular}{|l|}
0.5240 \\
\end{tabular} & 0.0593 & \begin{tabular}{|l|}
0.1269 \\
\end{tabular} & 0.8584 \\
\hline $\mathrm{NN}$ & 0.1291 & \begin{tabular}{|l|}
0.0699 \\
\end{tabular} & \begin{tabular}{|l|l|}
0.4856 \\
\end{tabular} & 0.0645 & \begin{tabular}{|l|l|}
0.1263 \\
\end{tabular} & 0.8448 \\
\hline Scene 7 & \multicolumn{3}{|c|}{$y^{1}$} & \multicolumn{3}{|c|}{$\overline{y^{2}}$} \\
\hline Model & NRMSE & MAE & $\mathrm{R}^{2}$ & NRMSE & \begin{tabular}{|l|} 
MAE \\
\end{tabular} & $\mathrm{R}^{2}$ \\
\hline GPR & 0.1082 & \begin{tabular}{|l|l|}
0.0604 \\
\end{tabular} & \begin{tabular}{|l|}
0.3958 \\
\end{tabular} & 0.0544 & 0.0815 & 0.9309 \\
\hline $\mathrm{NN}$ & 0.1049 & 0.0586 & 0.4062 & 0.0535 & \begin{tabular}{|l|l|}
0.0829 \\
\end{tabular} & 0.9361 \\
\hline Scene 8 & \multicolumn{3}{|c|}{$\overline{y^{1}}$} & \multicolumn{3}{|c|}{$\overline{y^{2}}$} \\
\hline Model & NRMSE & MAE & $\mathrm{R}^{2}$ & NRMSE & MAE & $\mathrm{R}^{2}$ \\
\hline GPR & 0.1126 & 0.0801 & 0.3428 & 0.0716 & \begin{tabular}{|l|}
0.1988 \\
\end{tabular} & 0.6483 \\
\hline $\mathrm{NN}$ & 0.1186 & 0.0845 & 0.3134 & 0.0730 & 0.2028 & 0.6319 \\
\hline
\end{tabular}

parameters from the RS2 quad-pol data (the true parameter maps), and the middle and bottom rows are the estimated outputs of the ML methods. It can be seen that both the NN and GPR models can estimate well the generated output features. Leads and /or open water, which are expected to appear smoother and with higher salinity, are represented with predicted high values for both $\mathrm{y}^{1}$ and $\mathrm{y}^{2}$.

The intensity images in Fig. 7 show some bright linefeatures in the south-east quarter of the image. The high intensity levels indicate high roughness which potentially can be narrow ridges. These features are clearly visible also in the surface roughness maps estimated by both the ML methods. The NN estimates a low $\mathrm{y}^{1}$ value, indicative of a smooth surface, while the GPR suggests high roughness. Hence, the output of the GPR model is in better correspondence with the intensity images. The optical image can unfortunately not be used to verify this particular observation, due to clouds and snow cover on the ice.

The advantageous uncertainty level property of the GPR model, allows for more detailed assessment of these estimates. Figure 9 shows the corresponding uncertainty maps for the $\mathrm{y}^{1}$ parameter for the two images presented in Fig. 6 and 8 (bottom-left panels). It can be observed that the assigned uncertainty is relative high for these features (Fig. 9 right). 

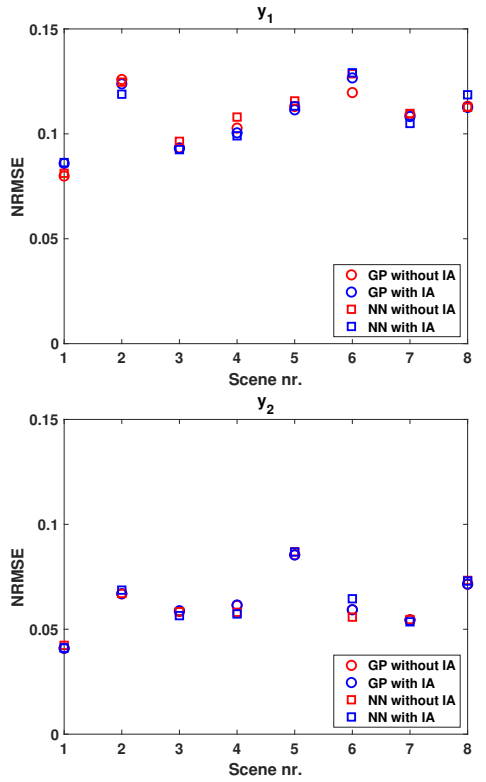
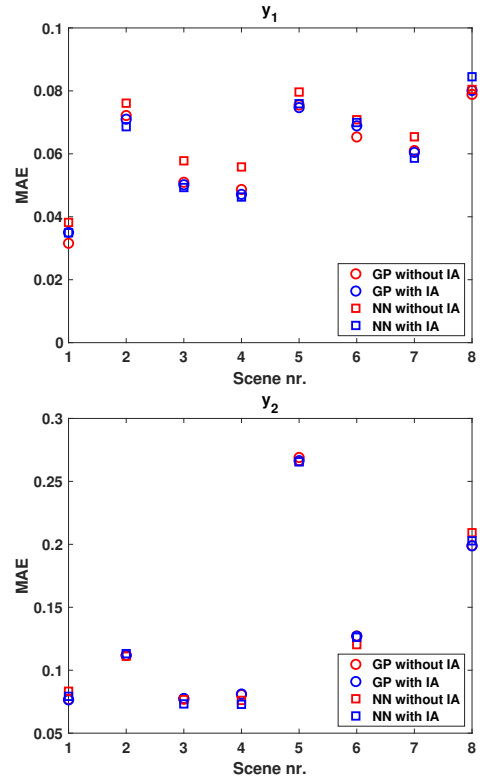
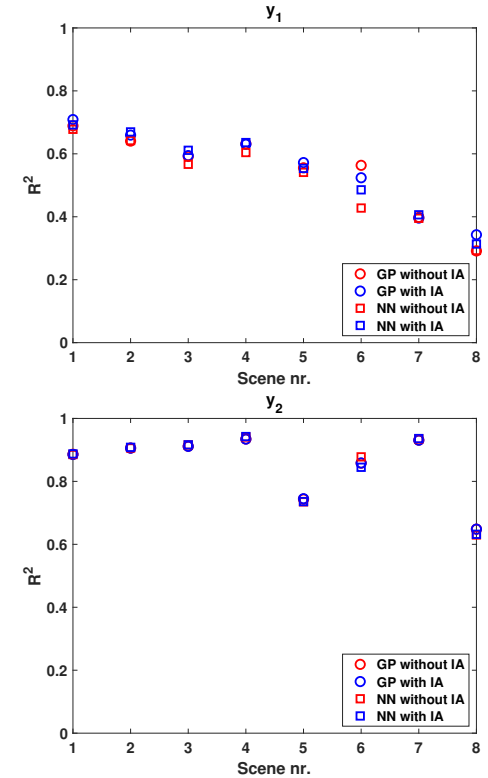

Fig. 4. The computed statistical measures for parameter $\mathrm{y}^{1}$ (top-row) and $\mathrm{y}^{2}$ (bottom-row): NRMSE (left), MAE (middle) and $\mathrm{R}^{2}$ (right). The plots correspond to the results presented in Table III and IV.

This indicates that the area with these features is not well represented in the input training data. It is also observed that the uncertainty level map of $\mathrm{y}^{1}$ for the scene 25/08/2016 shows relative high uncertainty for areas with presumably open water/leads (Fig. 9 left panel). These low backscattering areas may be highly contaminated by system noise, since the backscatter intensities in these dark patches are $-18 \pm 2 \mathrm{~dB}$ (for $\mathrm{HH}$ ) and $-28 \pm 2 \mathrm{~dB}$ (for HV)(see intensity images in Figs. 5 and 7). The noise floor (NESZ) of $\mathrm{S} 1$ is $<-23 \mathrm{~dB}$ (see Table I), and these areas are therefore very close to the noise floor, especially for the HV channel, which are used for some of the input features. Further investigations regarding the impact of system noise when predicting the quad-pol features $y^{1}$ and $y^{2}$ are therefore encouraged.

\section{B. Up-scaling of Sentinel-1 scenes}

We used the data acquired at 03/09/2016 (as in [39]) to show how the presented methodology can be applied for practical purposes. Figures 10 and 11 show the result of applying the trained GPR-model to perform spatial up-scaling of two quadpol parameters to the entire S1 scene. The top panel in Fig. 10 shows an optical S2 image overlapping with the RS2 and S1 scenes. The position of the swath of RS2 is shown as the red square and $\mathrm{S} 1$ is the black square. The HH intensity image of the large $\mathrm{S} 1$ scene can be seen in the bottom row.

Both the optical image and the S1 intensity image reveal a significant area of open water. For this area, the estimated output parameters predict high $\mathrm{y}^{1}$ values i.e smooth surface (top row in Fig. 11), and high $\mathrm{y}^{2}$ values i.e. salinity (bottom in Fig. 11). In fact, the estimated $y^{2}$ parameter shows an area with very high $\mathrm{y}^{2}$ values, which would be consistent with a mixing of thin ice/ film and open water. The rougher ridges discussed in the previous section, appear here in the large scene as well.
The results of the up-scaling show good correspondence with the interpretation made from the optical image and the $\mathrm{HH}$ intensity image.

\section{Limitations}

The here presented methodology has several limitations. The trained ML models are based on the assumption that the training data represents all sea ice conditions in order to make good predictions. However, it is well-known that the Arctic may show a large span of variations in sea ice conditions, making it challenging to build up a representative training data set for all situations. In this work we used melt-season images. This means that the snow cover is thin, wet and there might be frozen/ open melt ponds on the ice. Hence in practical use, the ML models may fail in areas with conditions differing from those picked for training the models. This will though be reflected in the certainty level map of GPR estimates. This would require to adjust the training data for freeze-up, winter and early-melt seasons for operational purposes.

The hyper-parameters in the ML models were tuned to this particular data set. Hence, deviations from this data might require a larger or lesser degree of smoothing, and therefore re-optimization of the model parameters.

The preprocessing prior to training and testing could also have an impact on the final products. For this study, the Radarsat-2 and Sentinel-1 products were projected to a common spatial grid covering approximately $50 \times 50 \mathrm{~m}$ on the ground. If a coarser resolution were selected then the detailed sea ice structure might be compromised, and with more smoothing, the accuracy in the up-scaling might improve due to less details being reconstructed. Hence, the choice of the resolution in the preprocessing should be selected based on the application. If a different size of the grid in the projection is used then new training and testing are encouraged. 

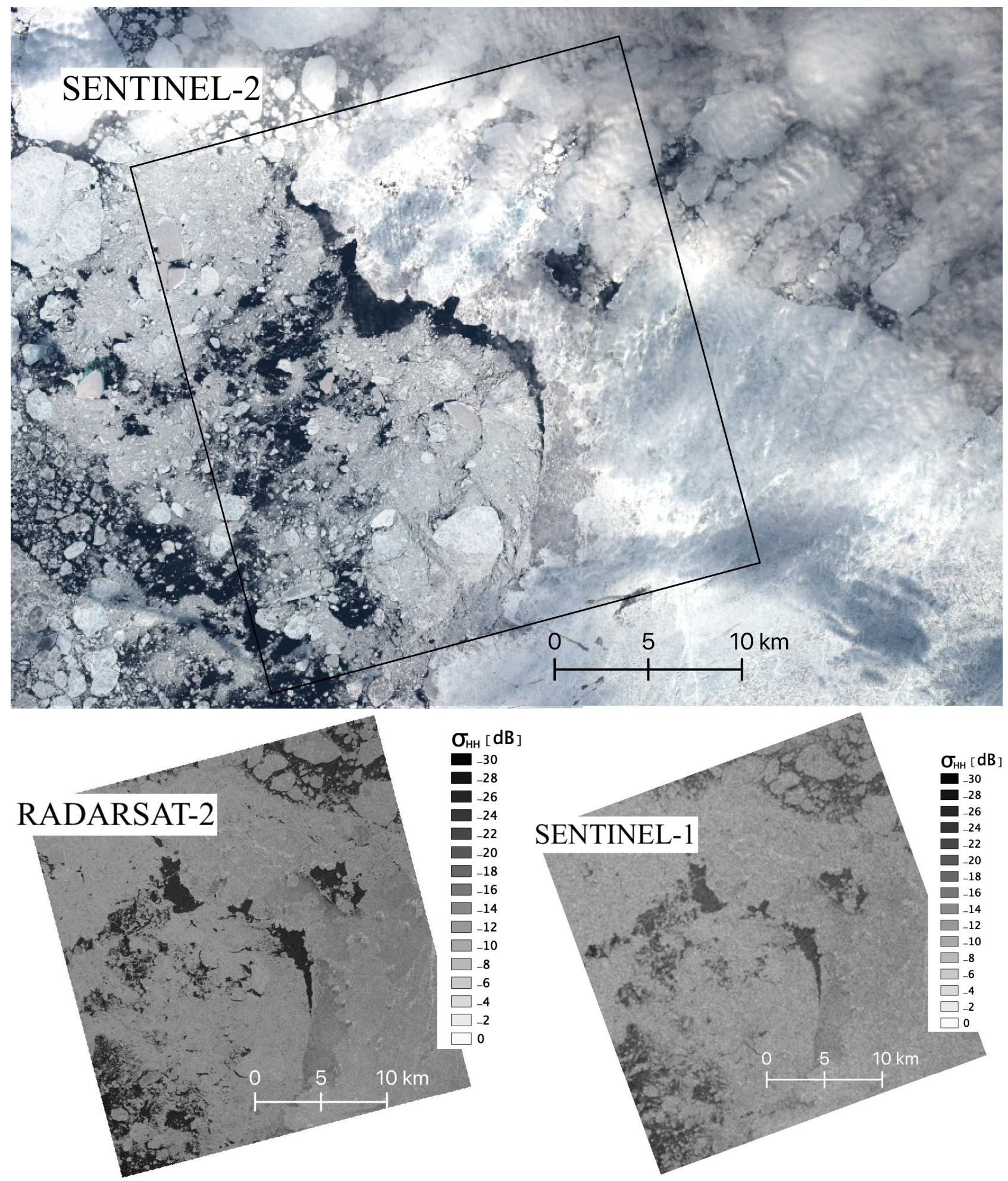

Fig. 5. Scene 25/08/2016: Sentinel-2 RGB image acquired 14:36 UTC (3 h before the Sentinel-1 image) (top row). The HH-intensity values $\left(\sigma_{H}^{0}{ }_{H}(d B)\right.$ ) (bottom row).

Another issue is the noise problem of S1. The standard S1 data product is known to have a severe noise problem, which displays itself as a strong banding in the range direction. This is associated with additive thermal system noise, and results from an inaccurate correction for the sub-swaths antenna beam patterns [47]. It varies with IA, and is most noticeable in the HV channel in low back-scattering areas. The impact of noise on the ML models should be further addressed in future work. This could be done by either developing specific preprocessing noise removal techniques or in fact training specific 

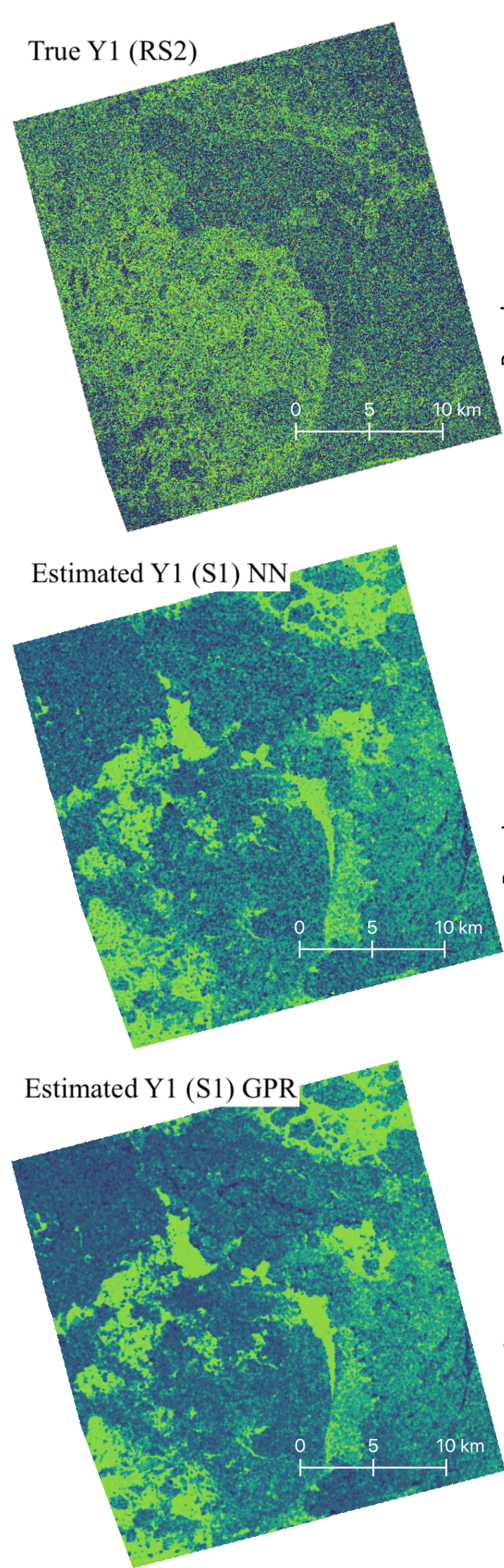
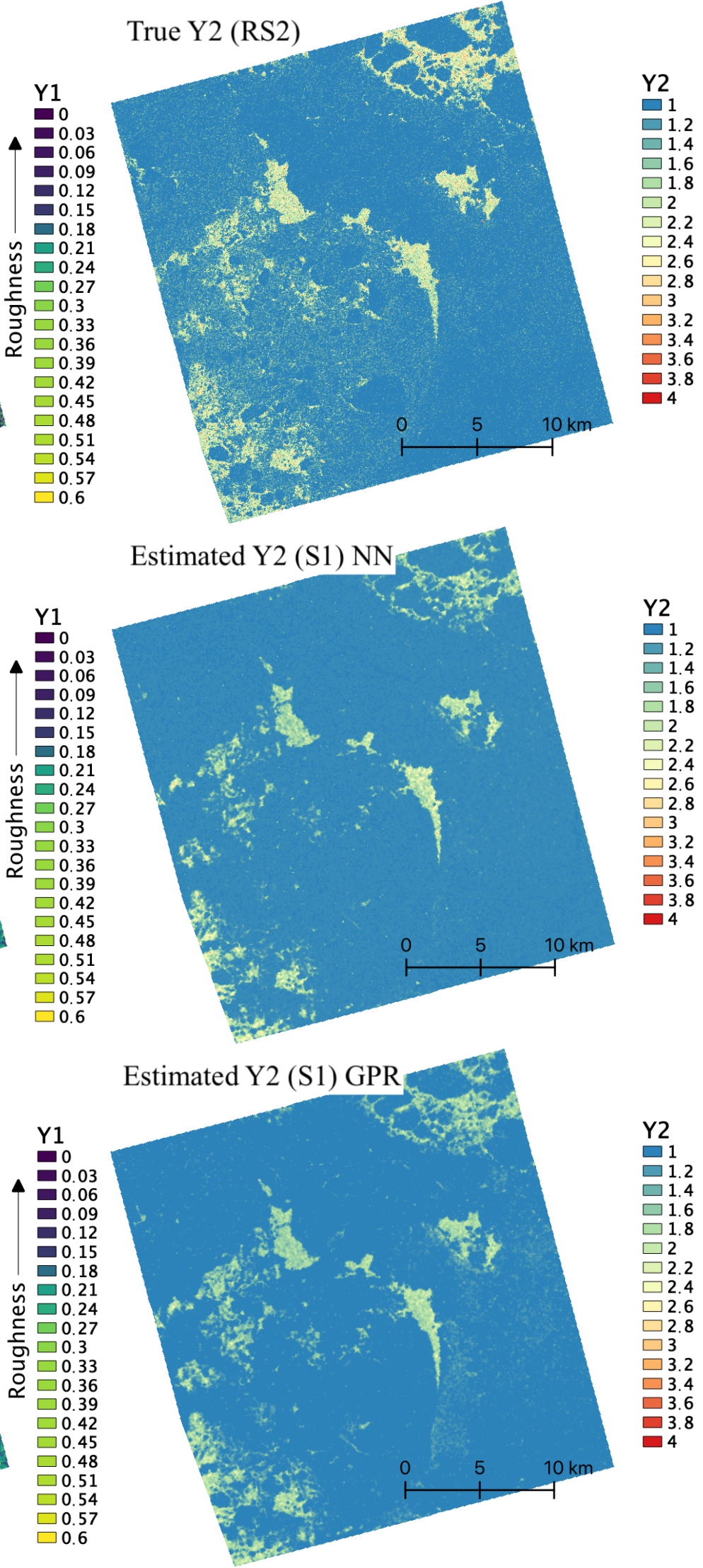

Fig. 6. Scene 25/08/2016: The output features $y^{1}$ (left column) and $y^{2}$ (right-column) for the ground truth (top row), the NN (middle row) and GPR (bottom row) models, when IA was not included as an input feature. 


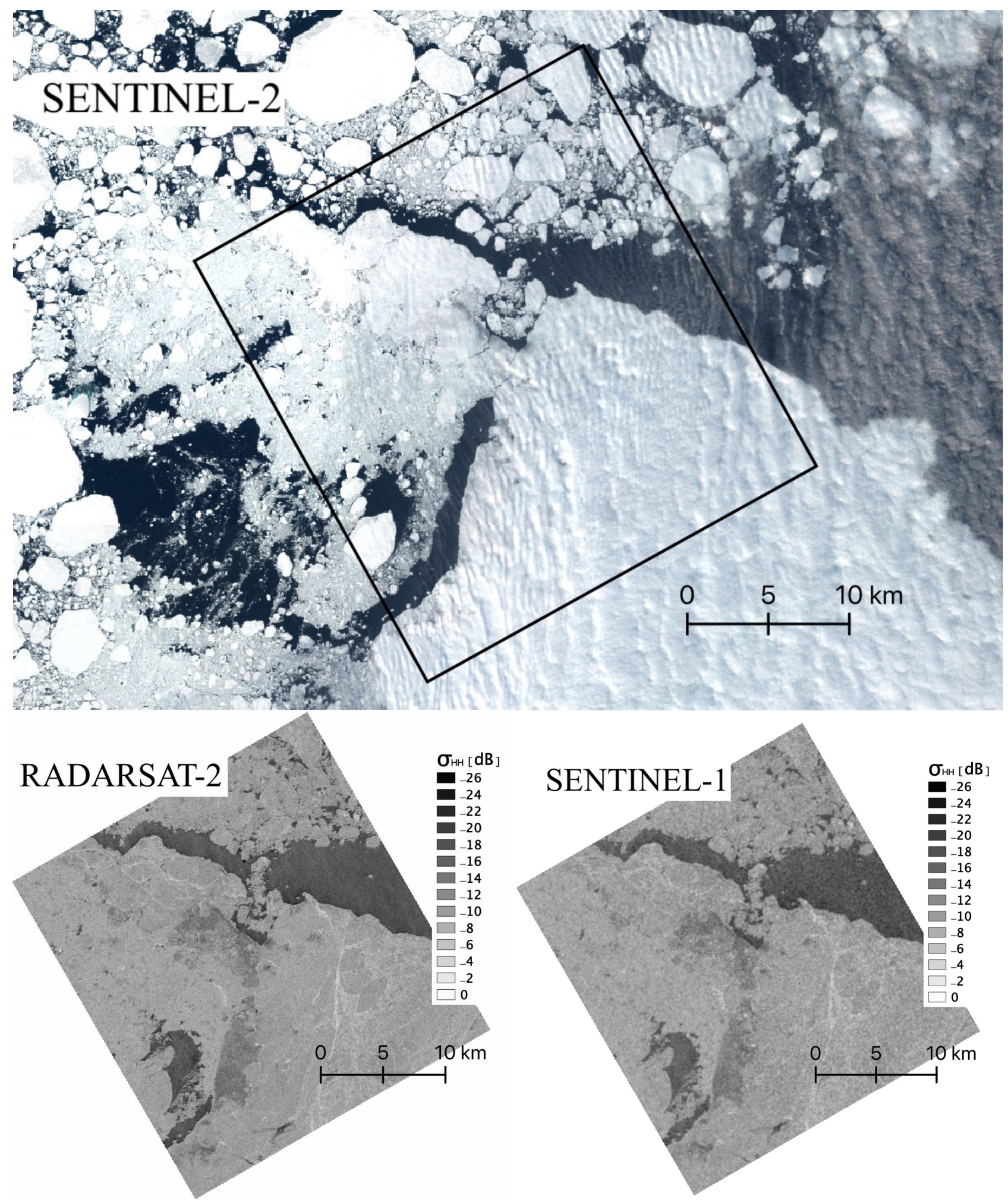

Fig. 7. Scene 03/09/2016: Sentinel-2 RGB image acquired 14:36 UTC ( $3 \mathrm{~h}$ before the Sentinel-1 image) (top row). The HH-intensity values $\left(\sigma_{H H}^{0}(d B)\right)$ (bottom row).

ML methods to cope with the noise problem.

\section{Vi. CONCLUSiOnS}

In this work, we presented a novel approach of using two well acknowledged ML methods, namely the GPR and
NN models, for improving geophysical information extraction from dual-pol SAR data. The overall goal was to explore the feasibility of increasing the information retrieval capacity of freely available, wide swaths dual-pol systems, such as $\mathrm{S} 1$, with regard to sea ice monitoring by exploiting coincident 

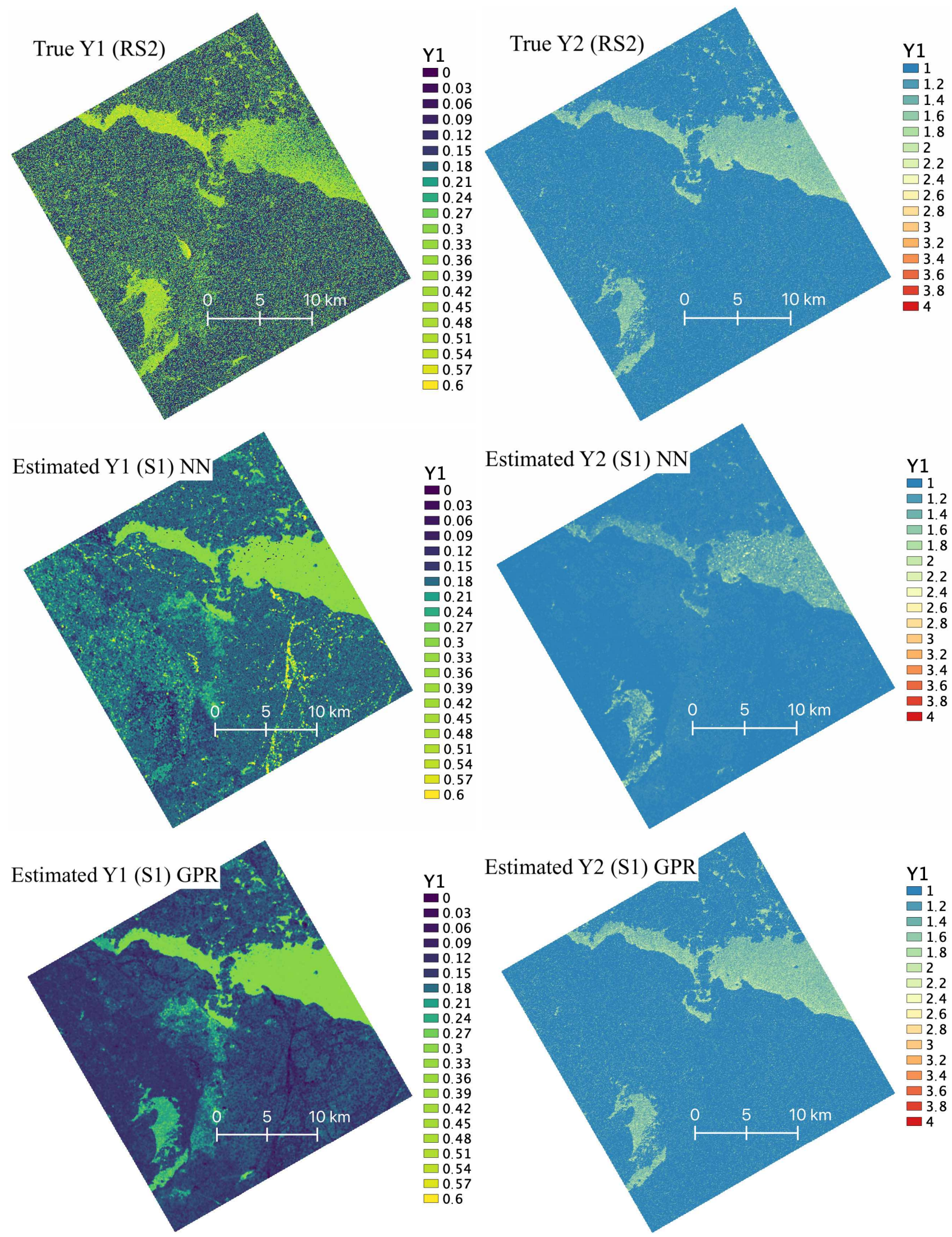

Fig. 8. Scene 03/09/2016: The output features $y^{1}$ (left column) and $y^{2}$ (right-column) for the ground truth (top row), the NN (middle row) and GPR (bottom row) models, when IA was not included as an input feature. 

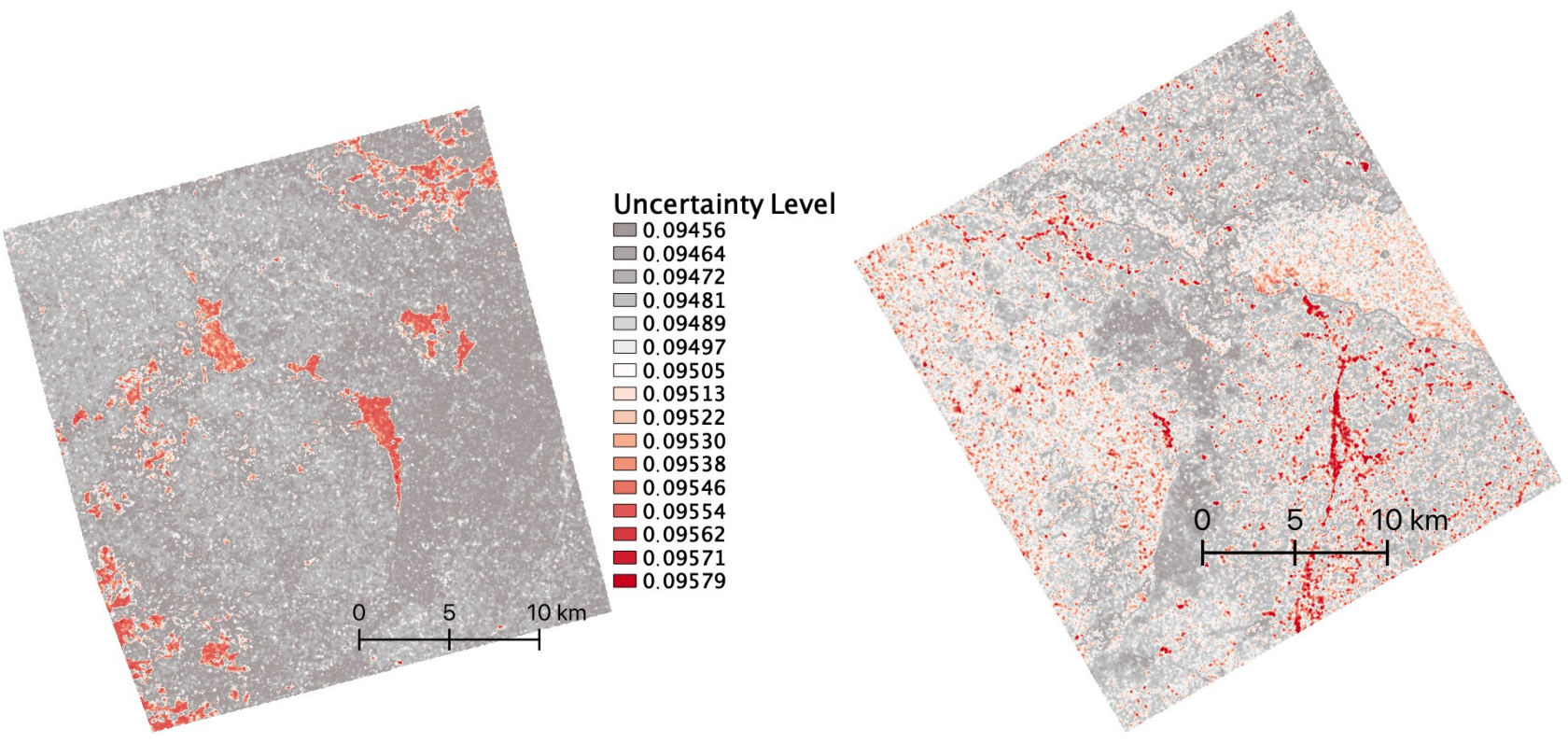

Fig. 9. Uncertainty level of the GPR for $\mathrm{y}^{1}$ for scene 25/08/2016 (left) and 03/09/2016 (right). The red colour indicates areas with relative high uncertainty.

quad and dual-pol observations. We focused on information associated to surface roughness and salinity, which are considered important for sea ice classification.

The ML models were trained to learn the functional relationship between two quad-pol output parameters and a corresponding input data matrix consisting of five/six input features, obtained from dual-pol data. More specifically, the predicted output parameters were the circular correlation coefficient $\left(\mathrm{y}^{1}\right)$, which has previously been found to be correlated with surface roughness, and the linear co-pol power ratio, which is strongly associated to dielectric properties $\left(\mathrm{y}^{2}\right)$, whereas the input parameters are constructed from the dual-pol polarimetric covariance matrix.

We trained the ML methods on eight overlapping scenes acquired by the quad-pol RS2 and dual-pol S1 systems. Subsequently, we performed two regression experiments. In the first experiment, we excluded IA from the input features, while in the second one IA was included. All the scenes were acquired between July and September, and further training and testing on scenes during the other seasons like the freeze-up, winter, and early-melt seasons are necessary.

Our results indicated that including IA has not shown significant improvement in the computed statistical regression performance measures. Our findings also showed differences in the prediction accuracy of the two parameters. The output parameter $\mathrm{y}^{2}$ can be better explained by the selected input features than $\mathrm{y}^{1}$. This is perhaps not surprising, as the only unknown observation for $\mathrm{y}^{2}$ is $\mid\left\langle\left. S_{V V}\right|^{2}\right\rangle$, which would be expected to be strongly correlated to at least one of the inputs, namely $\left\langle\left|S_{H H}\right|^{2}\right\rangle$.

Both ML approaches showed strong performance for these tasks. The NN model is computationally less expensive, however, the GPR model provides the uncertainty level of the estimates. Therefore, the choice of the particular method is user specific with regard to the priority of speed versus uncertainty information.
We also performed an experiment where the GPR-method was used for up-scaling the prediction of the two quad-pol parameters on an entire S1 scene. For this scene we also had an overlapping optical image available. This helped to interpret the estimates. Based on this study, we can conclude that the up-scaled parameter values seemed to be realistic and the experiment showed that the approach may have the potential to improve sea ice monitoring from dual-pol data. The presented approach have shown interesting capabilities, and may open new avenues for future use of dual-pol S1 data in sea ice monitoring. However, more extensive examinations are needed. In future work, new aspects of the usability of the methodology will be further studied. This includes a thorough investigation of the added value of using ML-estimates of $\mathrm{y}^{1}$ and $\mathrm{y}^{2}$ based on dual-pol data in large scale sea ice classification.

\section{ACKNOWLEDGMENT}

The Radarsat-2 and data Products (C)MDA LTD. 2015, 2016, and 2017, deliver by KSAT under the NorwegianCanadian Radarsat agreement. Sentinel-1 and Sentinel-2 products are from the European Space Agency (ESA). This work is funded by CIRFA through the RCN (research grant no. 237906).

The authors would like to thank Malin Johansson at UiT The Arctic University of Norway for her useful comments and suggestions.

\section{REFERENCES}

[1] J. Stroeve and D. Notz, "Changing state of arctic sea ice across all seasons," Environmental Research Letters, vol. 13, no. 10, p. 103001, sep 2018.

[2] N. Zakhvatkina, V. Smirnov, and I. Bychkova, "Satellite sar data-based sea ice classification: An overview," Geosciences, vol. 9, no. 1, 2019.

[3] J.-S. Lee and E. Pottier, Polarimetric Radar Imaging: From Basics to Applications, ser. Optical Science and Engineering. Boca Raton, USA: CRC Press, 2009, no. 143. 


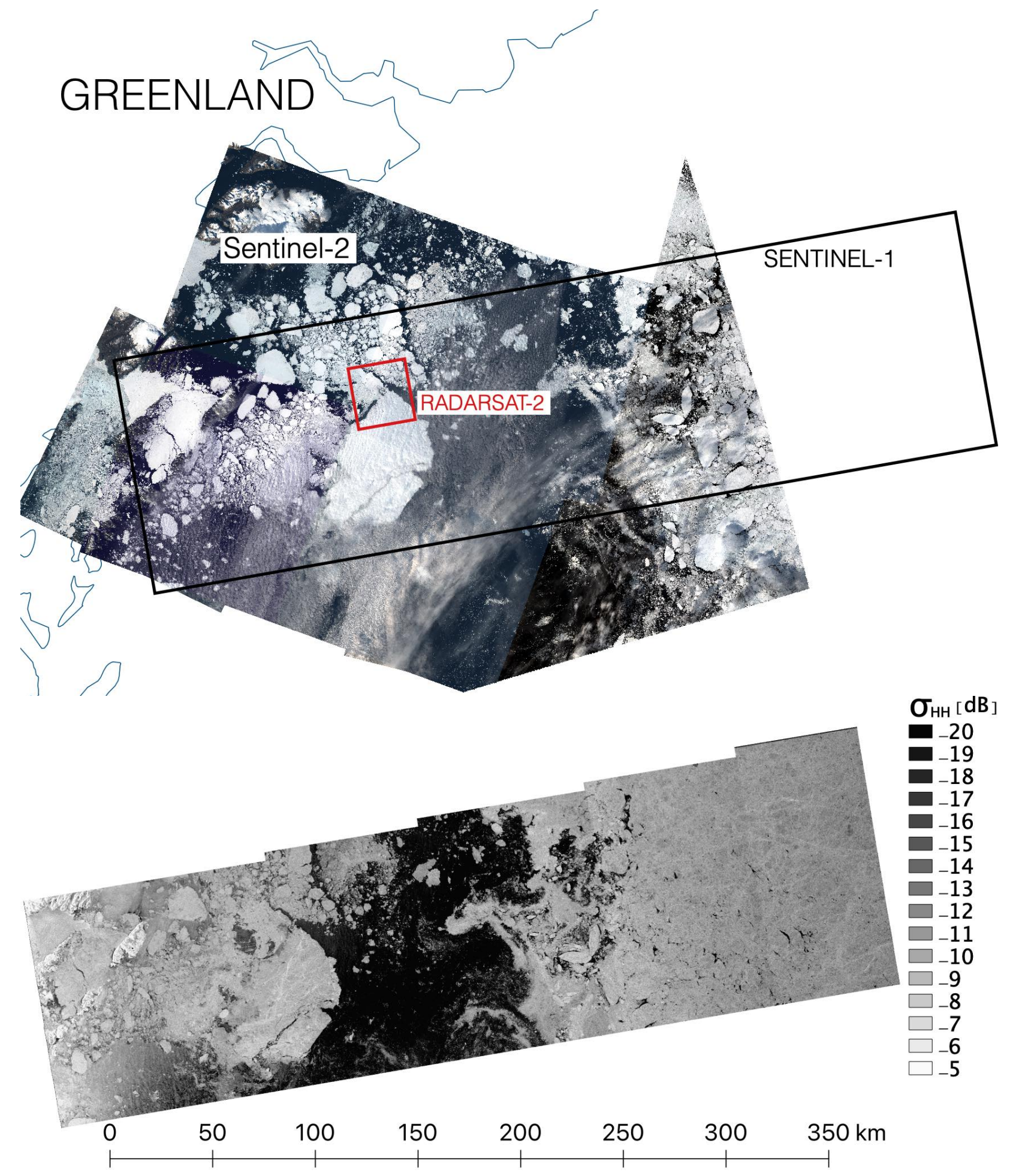

Fig. 10. Scene 03/09/2016: the overlapping S1, R2 and S2 scenes (top) and HH intensity image (bottom).

[4] S. R. Cloude, Polarisation: Applications in Remote Sensing. Oxford, UK: Oxford University Press, 2010.

[5] J. Holloway and K. Mengersen, "Statistical machine learning methods and remote sensing for sustainable development goals: A review," Remote Sensing, vol. 10, no. 9, 2018.

[6] L. Wang, J. Yan, and Y. Ma, Cloud Computing in Remote Sensing. London, UK: Chapman and Hall/CRCl, 2019.
[7] M. R. Drinkwater, R. Kwok, E. Rignot, H. Israelsson, R. G. Onstott, and D. P. Winebrenner, "Potential applications of polarimetry to the classification of sea ice," in Microwave remote sensing of sea ice, ser. Geophysical Monograph 68. American Geophysical Union, 1992.

[8] S. Cloude, D. Goodenough, and H. Chen, "Compact Decomposition Theory," IEEE Trans. Geosci. Remote Sens., vol. 9, no. 1, pp. 28-32, Jan. 2012. 


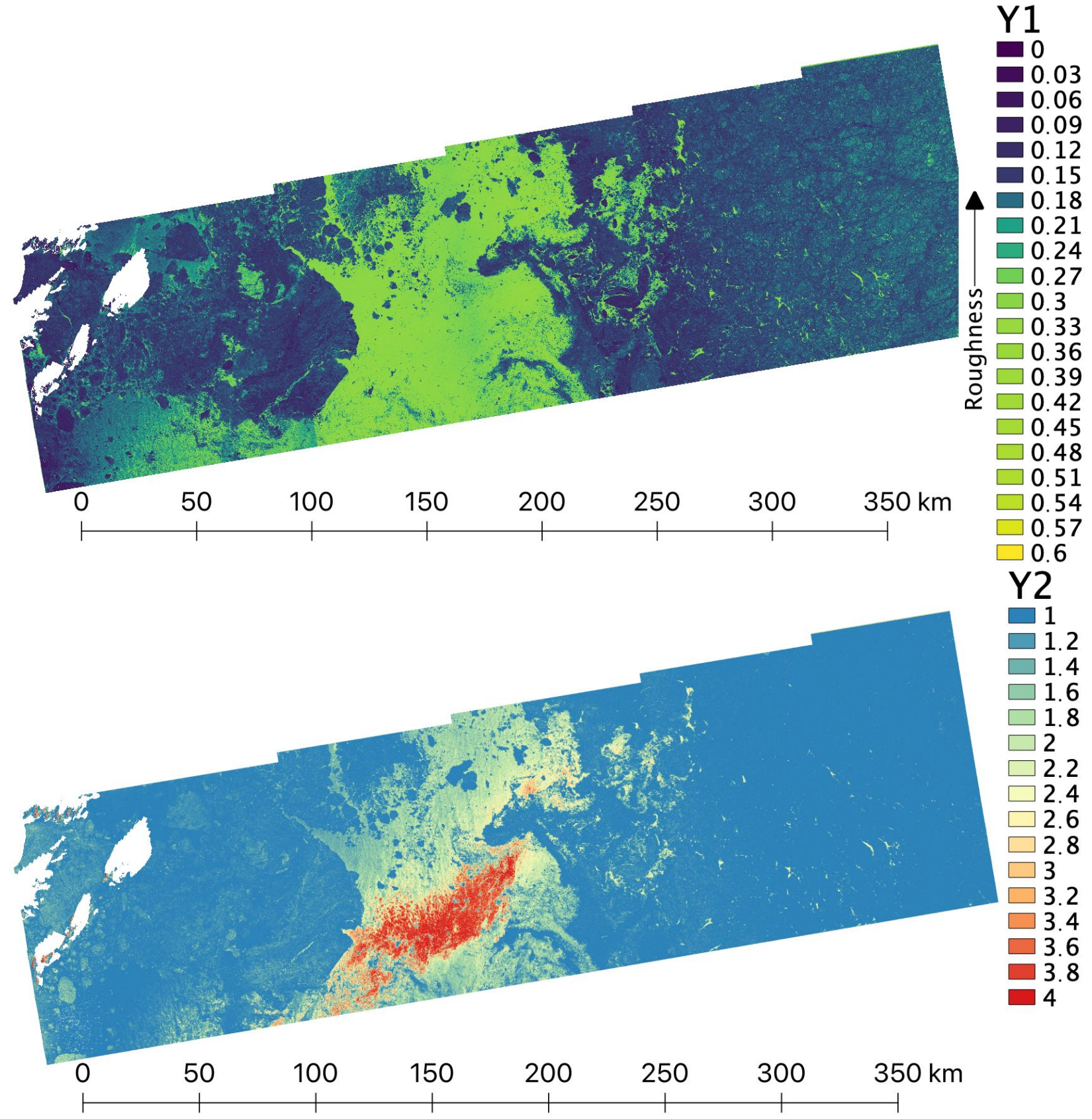

Fig. 11. Scene 03/09/2016: the up-scaled images of the $y^{1}$ (top) and $y^{2}$ (bottom) parameters using the GPR model.

[9] J.-S. Lee, M. R. Grunes, and R. Kwok, "Classification of multi-look polarimetric SAR imagery based on complex Wishart distribution," Int. J. Remote Sens., vol. 15, no. 11, pp. 2299-2311, Jul. 1994.

[10] A. P. Doulgeris, S. N. Anfinsen, and T. Eltoft, "Classification with a non-Gaussian model for PolSAR data," vol. 46, no. 10, pp. 2999-3009, Oct. 2008.

[11] _ - "Automated non-Gaussian clustering of polarimetric synthetic aperture radar images," vol. 49, no. 10, pp. 3665-3676, Oct. 2011.

[12] A. Iodice, A. Natale, and D. Riccio, "Retrieval of soil surface parameters via a polarimetric two-scale model," IEEE Trans. Geosci. Remote Sens., vol. 49, no. 7, pp. 2531-2547, Jul. 2011.

[13] S. V. Nghiem, R. Kwok, S. H. Yueh, and M. R. Drinkwater, "Polarimetric signatures of sea ice. 1: Theoretical model," Journal of Geophysical Research, Atmospheres, vol. 100, no. 7, pp. 13665-13679, 1995.

[14] — "Polarimetric signatures of sea ice: 2. experimental observations," Journal of Geophysical Research, Atmospheres, vol. 100, no. 7, pp
13681-13 698, 1995.

[15] W. Dierking, "Sea ice monitoring by synthetic aperture radar," Oceanography, vol. 26, no. 10, pp. 100-111, 2010.

[16] M. Johansson, J. King, A. P. Doulgeris, S. Gerland, S. Singha, G. Spreen, and T. Busche, "Combined observations of arctic sea ice with nearcoincident colocated X-band, C-band, and L-band SAR satellite remote sensing and helicopter-borne measurements," Journal of Geophysical Research, Oceans, vol. 122, no. 12, Jul. 2016.

[17] Y. Hara, R. G. Atkins, R. T. Shin, Jin Au Kong, S. H. Yueh, and R. Kwok, "Application of neural networks for sea ice classification in polarimetric sar images," IEEE Transactions on Geoscience and Remote Sensing, vol. 33, no. 3, pp. 740-748, 1995.

[18] N. Y. Zakhvatkina, V. Y. Alexandrov, O. M. Johannessen, S. Sandven, and I. Y. Frolov, "Classification of sea ice types in envisat synthetic aperture radar images," IEEE Transactions on Geoscience and Remote Sensing, vol. 51, no. 5, pp. 2587-2600, 2013 
[19] R. Ressel, A. Frost, and S. Lehner, "A neural network-based classification for sea ice types on x-band sar images," IEEE Journal of Selected Topics in Applied Earth Observations and Remote Sensing, vol. 8, no. 7, pp. 3672-3680, 2015.

[20] S. Leigh, Z. Wang, and D. A. Clausi, "Automated ice-water classification using dual polarization sar satellite imagery," IEEE Transactions on Geoscience and Remote Sensing, vol. 52, no. 9, pp. 5529-5539, 2014.

[21] M.-A. N. Moen, A. P. Doulgeris, S. N. Anfinsen, A. H. H. Renner, N. Hughes, S. Gerland, and T. Eltoft, "Comparison of feature based segmentation of full polarimetric sar satellite sea ice images with manually drawn ice charts," The Cryosphere, vol. 7, no. 6, pp. 1693-1705, 2013. [Online]. Available: https://www.thecryosphere.net/7/1693/2013/

[22] J. Lohse, A. P. Dougleris, and W. Dierking, "An optimal decision-tree design strategy and its application to sea ice classification from sar imagery," Remote Sensing, vol. 11 (13), p. 1574, 2019.

[23] K. Blix, M. M. Espeseth, and T. Eltoft, "Machine learning simulations of quad-polarimteric features from dual-polarimetric measurements over sea ice," in 12th European Conference on Synthetic Aperture Radar, Electronic proceedings, 2018, pp. 661-665.

[24] — "Up-scaling from quad-polarimetric to dual-polarimetric sar data using machine learning gaussian process regression," in 2018 IEEE International Geoscience and Remote Sensing Symposium, IGARSS 2018, Valencia, Spain, 2018.

[25] _ "Comparision of machine learning methods for predicting quadpolarimetric parameters from dual-polarimetric sar data," in 2020 IEEE International Geoscience and Remote Sensing Symposium, IGARSS 2020, Waikoloa, Hawaii, USA, 2020, 2020, p. in review.

[26] J. Verrelst, J. Muñoz, L. Alonso, J. P. Rivera, G. Camps-Valls, and J. Moreno, "Machine learning regression algorithms for biophysical parameter retrieval: Opportunities for sentinel-2 and -3," Remote Sensing of Environment, vol. 118, pp. 127-139, 2012.

[27] K. Blix, G. Camps-Valls, and R. Jenssen, "Gaussian process sensitivity analysis for oceanic chlorophyll estimation," IEEE Journal of Selected Topics in Applied Earth Observations and Remote Sensing, vol. 10 (4), pp. 1265-1277, April 2017.

[28] K. Blix and T. Eltoft, "Evaluation of feature ranking and regression methods for oceanic chlorophyll-a estimation," IEEE Journal of Selected Topics in Applied Earth Observations and Remote Sensing, vol. 11 (5), pp. 1403-1418, 2018.

[29] W. Gregory, M. Tsamados, J. Stroeve, and P. Sollich, "Regional september sea ice forecasting with complex networks and gaussian processes," Weather and Forecasting, 2020. [Online]. Available: https://doi.org/10.1175/WAF-D-19-0107.1

[30] H. Wakabayashi, T. Matsuoka, K. Nakamura, and F. Nishio, "Polarimetric characteristics of sea ice in the sea of Okhotsk observed by airborne L-band sar," IEEE Transactions on Geoscience and Remote Sensing, vol. 42, no. 11, 2004, 2412-2425.

[31] T. Geldsetzer and J. J. Yackel, "Sea ice type and open water discrimination using dual co-polarized c-band sar," Candian Journal of Remote Sensing, vol. 35, no. 1, pp. 73-84, 2014.

[32] P. Yang, L. Guo, and Q. Wang, "Circularly polarized wave scattering from two-dimensional dielectric rough sea surface," Progress in Electromagnetism Research, vol. 44, pp. 119-126, 2015.

[33] F. Mattia, T. L. Toan, J. C. Souyris, G. D. Carolis, N. Floury, F. Posa and G. Pasquariello, "The effect of surface roughness on multi-frequency polarimetric sar data," IEEE Transactions on Geoscience and Remote Sensing, vol. 35, no. 7, 1997, 954-966.

[34] D. L. Schuler, J.-S. Lee, and D. Kasilingam, "Surface roughness and slope measurements using polarimetric sar data," IEEE Transactions on Geoscience and Remote Sensing, vol. 40, no. 3, 2002, 687-697.

[35] T. L. Ainsworth, D. L. Schuler, and J.-S. Lee, "Polarimetric sar characterization of man-made structures in urban areas using normalized circular-pol correlation coefficients," Remote Sensing of Environment, vol. 112, pp. 2876-2885, 2008.

[36] G. R. Valenzuela, "Theories for the interaction of electromagnetic and oceanic waves - a review," Boundary-Layer Meteorology, no. 1-4, pp. 61-85, Jan. 1978.

[37] D. Winebrenner, "Accuracy of thin ice/open water classification using multi-polarization sar," in Proc. IEEE Int. Geosc. Remote Sens. Symp., IGARSS 2005, vol. 1, New York, 1990, pp. 2237-2240.

[38] Y. Oh, K. Sarabandi, and F. T. Ulaby, "An empirical model and an inversion technique for the scattering from soil surfaces," IEEE Transactions on Geoscience and Remote Sensing, vol. 30, no. 3, pp. 370-381-1104, 1992

[39] T. G. Yitayew, W. Dierking, D. V. Divine, T. Eltoft, L. Ferro-Famil, A. Rösel, and J. Negrel, "Validation of sea-ice topographic heights derived from tandem-x interferometric sar data with results from laser profiler and photogrammetry," IEEE Transactions on Geoscience and Remote Sensing, vol. 56, no. 11, pp. 6504-6520, 2018.

[40] M. Bourbigot, H. Johnsen, and R. Piantanida, "Sentinel-1 product definition," MPC-S1, Tech. Rep. S1-RS-MDA-52-7440, Issue 2/7, March 2016, online; accessed 14-June-2020. [Online]. Available: https://sentinel.esa.int/documents/247904/1877131/Sentinel1-Product-Definition

[41] "Radarsat-2 product description," MacDonald, Dettwiler and Associates Ltd., Rickmond, BC, USA, RN-SP-52-1238, vol. Issue 1/13, 2016.

[42] MacDonald, Dettwiler and Associates Ltd, "Radarsat-2 product description," 2014.

[43] M. S. Mahmud, T. Geldsetzer, S. E. L. Howell, J. J. Yackel, V. Nandan, and R. K. Scharien, "Incidence angle dependence of hh-polarized c- and l-band wintertime backscatter over arctic sea ice," IEEE Transactions on Geoscience and Remote Sensing, vol. 56, no. 11, pp. 6686-6698, 2018.

[44] A. S. Komarov and M. Buehner, "Detection of first-year and multi-year sea ice from dual-polarization sar images under cold conditions," IEEE Transactions on Geoscience and Remote Sensing, vol. 57, no. 11, pp. 9109-9123, 2019.

[45] J. J. Yackel, D. G. Barber, T. N. Papakyriakou, and C. Breneman, "Firstyear sea ice spring melt transitions in the canadian arctic archipelago from time-series synthetic aperture radar data, 1992-2002," Hydrological Processes, vol. 21, pp. 253-265, 2007.

[46] C. E. Rasmussen and C. K. I. Williams, Gaussian Processes for Machine Learning. The MIT Press, 2005.

[47] J.-W. Park, J.-S. Won, A. A. Korsov, M.Babiker, and N. Miranda, “Textural noise correction for sentinel-1 topsar cross-polarization channel images," IEEE Transactions on Geoscience and Remote Sensing, vol. 57, no. 6 , pp. $4040-4049,2019$. 


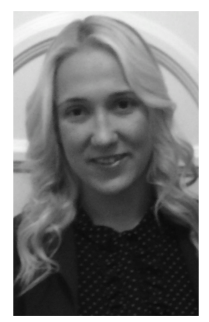

Katalin Blix received a B.Sc. degree in Geosciences (2010) from the Western Norway University of Applied Sciences, and a Civil engineer/ M.Sc. (2014) and Ph.D. (2019) degrees from the Department of Physics and Technology at the UiT the Arctic University of Norway. She is currently a researcher at the Centre for Integrated Remote Sensing and Forecasting for Arctic Operations (CIRFA), Department of Physics and Technology, UiT the Arctic University of Norway. Blix's research interests include machine learning algorithm development for regression, classification and feature relevance extraction; kernel machines, Bayesian statistics, and applications to remotely sensed data, such as biogeochemical and sea ice parameter retrieval. Blix is an active member of the Sentinel-3 validation team (since 2016) and Association of Polar Early Career Scientists (since 2019). She was the recipient of the 2017 Arctic Frontiers Outstanding Poster Award Overall Winner, the CIRFA 2017 Best Poster Award and the International Ocean Color Science (IOCS) 2019 travel grant awarded by EUMETSAT.

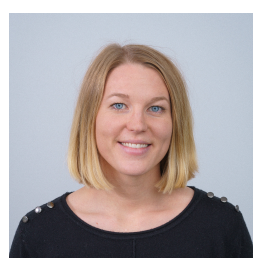

Martine M. Espeseth received the M.Sc. and Ph.D. degrees from the Department of Physics and Technology, UiT The Arctic University of Norway, Troms $\varnothing$, Norway, in 2015 and 2019, respectively.

She is currently a Postdoc at the same department, with the Centre for Integrated Remote Sensing and Forecasting for Arctic Operations (CIRFA). From February to April 2016 and August to December 2018, she was a Visiting Ph.D. student with the Jet Propulsion Laboratory, California Institute of Technology, Pasadena, CA, USA. Her current research interests include remote sensing of polarimetric SAR and with a focus on compact polarimetry within both marine oil pollution and sea ice applications.

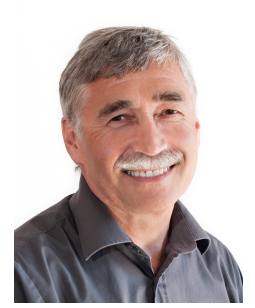

Torbjørn Eltoft (M'92) joined the Faculty of Science and Technology, UiT the Arctic University of Norway in 1988, where he is employed as a Professor in remote sensing at the Department of Physics and Technology. Eltoft is the Director of CIRFA (Centre for Integrated Remote Sensing and Forecasting for Arctic Operations), a Centre for Research-based awarded by the Norwegian Research Council in 2014. Eltoft's research interests include multi-dimensional signal and image analysis, statistical modelling, neural networks, and machine learning, with applications in multi-channel Synthetic Aperture Radar and ocean colour remote sensing. He served as Associate Editor of the Elsevier journal Pattern Recognition for the period 2005-2011, and he was guesteditor for the journal Remote Sensing on the Special Issue for the PolInSAR 2017 conference. Eltoft has a significant publication record in the area of signal processing and remote sensing, and he was the recipient of the year 2000 Outstanding Paper Award in Neural Networks awarded by IEEE Neural Networks Council, and of the Honourable Mention for the 2003 Pattern Recognition Journal Best Paper Award. Eltoft was the winner of the 2017 UiT Award for Research and Development. 